Revista Iberoamericana de las Ciencias Biológicas y Agropecuarias

\title{
Zeolitas nativas en el tratamiento de agua residual doméstica
}

\author{
Native Zeolites in Domestic Wastewater Treatment
}

\section{Zeólitas nativas no tratamento de águas residuais domésticas}

\begin{abstract}
Noemi Méndez de los Santos
Tecnológico Nacional de México, Instituto Tecnológico de Villahermosa, México mimilla6566@hotmail.com https://orcid.org/0000-0002-3788-8571

Gaspar López Ocaña Universidad Juárez Autónoma de Tabasco, México ocanagl77@hotmail.com https://orcid.org/0000-0002-8402-8160
\end{abstract}

\section{Resumen}

En esta investigación zeolitas de río y de cerro fueron evaluadas para ver su potencial en el tratamiento de aguas residuales. En primer lugar, se diseñó y construyó un sistema experimental de tres biorreactores de lecho fijo de flujo ascendente (BLFFA) con una altura de 1.5 metros y diámetro de cuatro pulgadas, seguidamente se evaluó el potencial de las zeolitas nativas y una testigo comercial en el tratamiento de aguas residuales. Se arrancaron los BLFFA con un diseño factorial $3 \times 3$, operando con zeolita de río, zeolita de cerro y zeolita comercial (control), todas con partículas de 1/4 de pulgada, utilizando tres diferentes alturas de lecho $(0.75,0.90$ y $1.10 \mathrm{~m})$ y tres tiempos de retención hidráulica $(6,12$ y 24 horas). Se evaluaron las variables de respuesta mediante un análisis de varianza multifactorial y para discriminar entre las medias se empleó el método de diferencia mínima significativa (LSD) de Fisher ( $\mathrm{p}<0.05,95 \%$ de confianza). La zeolita de cerró presentó (promedio, $N=10$ ) las 
Revista Iberoamericana de las Ciencias Biológicas y Agropecuarias

mejores características fisicoquímicas con densidad real y aparente de 2700 y $1470 \mathrm{~kg} / \mathrm{m}^{3}$ respectivamente, peso específico de $2240 \mathrm{~kg} / \mathrm{m}^{3}$, porosidad de $62 \%$, absorción de $15.20 \%$, solubilidad en ácido clorhídrico de $29.96 \%$, pH de 7.4 y conductividad eléctrica de 70 $\mathrm{mS} / \mathrm{cm}$. Al agua residual con las que se desarrollaron los tratamientos se le aplicó previo al experimento un tratamiento primario (rejilla, desarenador, desnatador y fosa séptica) y presentó valores (promedio, $N=9$ ) de temperatura de $25.26^{\circ} \mathrm{C}$, $\mathrm{pH}$ de 8.63 , sólidos disueltos totales con $950.50 \mathrm{mg} / \mathrm{L}$, color de 1305.10 UC, turbiedad 96.37 UNT y demanda química de oxígeno de $373 \mathrm{mg} / \mathrm{L}$. De las zeolitas evaluadas $(N=81)$ en el BLFFA, la que presentó el mejor tratamiento fue la zeolita de cerro operando con una altura de lecho de $0.9 \mathrm{~m}$ y con 24 horas de tiempo de retención hidráulica; obtuvo las mejores eficiencias de remoción con $71.42 \%$ en sólidos disueltos totales, $84.34 \%$ para turbiedad, $96.33 \%$ para color y $97.28 \%$ para la demanda química de oxígeno. La altura óptima en los BLFFA fue $0.9 \mathrm{~m}$, factor importante de evaluar, ya que a mayor altura se aumentan los costos y las eficiencias no son relevantes para justificar la inversión y a menor altura cae el rendimiento en calidad del agua tratada. En conclusión, las zeolitas nativas cuentan con propiedades idóneas para ser utilizadas en el tratamiento de agua residual doméstica y podemos recomendar la implementación de los BLFFA en el tratamiento secundario de efluentes domésticos en sistemas descentralizados del sureste de México como una alternativa viable en el tratamiento de sus aguas residuales domésticas.

Palabras clave: biorreactor de lecho fijo de flujo ascendente, demanda química de oxígeno, eficiencia de remoción, tiempo de retención hidráulica.

\section{Abstract}

Natural zeolites have a high purification capacity in wastewater due to their porosity, specific surface, cation exchange capacity and they are a product available in nature. In Tabasco, Mexico, river and hill zeolites were evaluated to see their potential in wastewater treatment. First, an experimental system of three upflow fixed-bed bioreactors (UFBB) with a height of 1.5 meters and diameter of 4 inches was designed and built, then the potential of native zeolites and a commercial control in the field were evaluated. sewage treatment. The UFBB were started with a $3 \times 3$ factorial design, operating with river zeolite, hill zeolite and commercial zeolite (control), all with $1 / 4$ inch particles, using three different bed heights $(0.75$, 
0.90 and $1.10 \mathrm{~m})$ and three times. hydraulic retention $(6,12$ and 24 hours). The response variables were evaluated by means of a multifactorial ANOVA and to discriminate between the means the Fisher's least significant difference (LSD) method ( $p<0.05,95 \%$ confidence) was used. The closed zeolite presented (average, $N=10$ ) the best physicochemical characteristics with are real and apparent density of 2700 and $1470 \mathrm{~kg} / \mathrm{m}^{3}$ respectively, specific weight $2240 \mathrm{~kg} / \mathrm{m}^{3}$, porosity of $62 \%$, absorption of $15.20 \%$, solubility in hydrochloric acid of $29.96 \%, \mathrm{pH}$ of 7.4 and electrical conductivity of $70 \mathrm{mS} / \mathrm{cm}$. The residual water with which the treatments were developed was applied prior to the experiment a primary treatment (grid, sand trap, skimmer and septic tank) and presented temperature values (average, $\mathrm{N}=9$ ) of $25.26^{\circ} \mathrm{C}, \mathrm{pH}$ of 8.63 , Total dissolved solids with $950.50 \mathrm{mg} / \mathrm{L}$, color of 1305.10 UC, turbidity 96.37 NTU and chemical oxygen demand of $373 \mathrm{mg} / \mathrm{L}$. Of the zeolites evaluated $(N=81)$ in the UFBB, the one that presented the best treatment was the hill zeolite operating with a bed height of $0.9 \mathrm{~m}$ and with 24 hours of hydraulic retention time, obtaining the best removal efficiencies with $71.42 \%$ in total dissolved solids, $84.34 \%$ for turbidity, $96.33 \%$ for color and $97.28 \%$ for the chemical oxygen demand, followed by the commercial zeolite that had very similar responses and finally the river zeolite treatment that also presents good results in comparison with other means of support. The optimal height in the UFBB was $0.9 \mathrm{~m}$, an important factor to evaluate since the higher the elevation costs are increased and the efficiencies are not relevant to justify the investment and the lower the performance in quality of the treated water falls. Finally, native zeolites have ideal properties to be used in the treatment of domestic wastewater and we can recommend the implementation of UFBB in the secondary treatment of domestic effluents in decentralized systems in the southeast of Mexico as a viable alternative in the treatment of their domestic wastewater.

Keywords: upflow fixed bed bioreactor, chemical oxygen demand, removal efficiency, hydraulic retention time. 
Revista Iberoamericana de las Ciencias Biológicas y Agropecuarias

\section{Resumo}

Nesta pesquisa, zeólitas de rios e morros foram avaliados para ver seu potencial no tratamento de águas residuais. En primer lugar, se diseñó y construyó un sistema experimental de tres biorreactores de lecho fijo de flujo ascendente (BLFFA) con una altura de 1.5 metros y diámetro de cuatro pulgadas, seguidamente se evaluó el potencial de las zeolitas nativas y una testigo comercial en el tratamento de águas residuais. Os BLFFAs foram depenados em esquema fatorial $3 \times 3$, operando com zeólita de rio, zeólita de colina e zeólita comercial (controle), todos com partículas de 1/4 de polegada, usando três alturas de leito diferentes $(0,75,0,90$ e $1,10 \mathrm{~m})$. vezes $(6,12$ e 24 horas). As variáveis de resposta foram avaliadas por meio de uma análise de variância multifatorial e o método da diferença mínima significativa de Fisher (LSD) foi usado para discriminar as médias ( $p<0,05,95 \%$ de confiança). A zeólita fechada apresentou (média, $\mathrm{N}=10$ ) as melhores características físico-químicas com densidade real e aparente de 2700 e $1470 \mathrm{~kg} / \mathrm{m} 3$ respectivamente, peso específico de 2240 $\mathrm{kg} / \mathrm{m}^{3}$, porosidade de $62 \%$, absorção de $15,20 \%$, solubilidade em clorídrico ácido de $29,96 \%$, pH de 7,4 e condutividade elétrica de $70 \mathrm{mS} / \mathrm{cm}$. A água residual com a qual os tratamentos foram desenvolvidos foi submetido a um tratamento primário prévio ao experimento (grade, caixa de areia, skimmer e fossa séptica) e apresentou valores de temperatura (média, $\mathrm{N}=9$ ) de $25,26^{\circ} \mathrm{C}, \mathrm{pH}$ de 8,63 , Total de sólidos dissolvidos com 950,50 mg / L, cor de 1305,10 UC, turbidez 96,37 NTU e demanda química de oxigênio de $373 \mathrm{mg} / \mathrm{L}$. Das zeólitas avaliadas ( $=81)$ no BLFFA, a que apresentou melhor tratamento foi a zeólita de morro operando com altura de leito de 0,9 me com tempo de retenção hidráulica de 24 horas; obtiveram as melhores eficiências de remoção com 71,42\% no total de sólidos dissolvidos, 84,34\% para turbidez, 96,33\% para cor e 97,28\% para demanda química de oxigênio. A altura ótima no BLFFA foi de $0,9 \mathrm{~m}$, fator importante a ser avaliado, visto que quanto maiores os custos de elevação aumentam e as eficiências não são relevantes para justificar o investimento, e menor o desempenho em qualidade das quedas d'água tratadas. Concluindo, zeólitas nativas possuem propriedades ideais para serem utilizadas no tratamento de águas residuais domésticas e podemos recomendar a implementação do BLFFA no tratamento secundário de efluentes domésticos em sistemas descentralizados no sudeste do México como uma alternativa viável no tratamento de suas águas residuais domésticas. 
Palavras-chave: biorreator de leito fixo de fluxo ascendente, demanda química de oxigênio, eficiência de remoção, tempo de retenção hidráulica.

Fecha recepción: Julio 2020

Fecha aceptación: Diciembre 2020

\section{Introducción}

El tratamiento de aguas residuales tiene como fin la depuración de contaminantes utilizando operaciones y procesos unitarios mediante una combinación capaz de sanear las aguas residuales para poder reutilizarlas en actividades diversas (Noyola, MorganSagastume, Güereca, 2013). En el mundo, más de $80 \%$ de las aguas residuales son vertidas sin tratamiento alguno, según una estimación frecuentemente citada (Organización de las Naciones Unidas para la Educación, la Ciencia y la Cultura [Unesco], 2017). Esto evidentemente tiene repercusiones negativas en la salud humana, la productividad económica, la calidad de los recursos de agua dulce y los ecosistemas (Unesco, 2017).

Existen varios niveles para tratar las aguas residuales (pretratamiento, tratamiento primario, tratamiento secundario, tratamiento avanzado y especiales). Los más estudiados son los secundarios (biológicos). Este tipo de tratamiento es fundamental en el desarrollo de trenes de tratamiento de aguas residuales por tiempos de construcción, operación y mantenimiento (Comisión Nacional del Agua [Conagua], 2016a). Una de las tecnologías biológicas que ofrece bajos costos de operación, mantenimiento, reducción de energía y producción de lodos son los reactores de lecho empacado: operan de manera eficiente por el medio de soporte utilizado, logran una óptima adherencia de los microorganismos y proporcionan la máxima área superficial al flujo del líquido bajo tratamiento, pudiendo ser aerobios o anaerobios (Ganesh, Rajinikanth, Thanikal, Ramanujam y Torrijos, 2010). Por otro lado, el tratamiento de aguas residuales con tecnologías sostenibles se fundamenta en procedimientos naturales que no utilizan aditivos químicos y aprovechan los recursos de las zonas donde se generan. Además, debido a que la eliminación de las sustancias contaminantes en el agua se logra aprovechando la vegetación acuática, el suelo, materiales locales o microorganismos, la inversión inicial y el mantenimiento de este tipo de sistema resultan más económicos en comparación con otras opciones (Morató, Pires y Subirana, 2009). 
Revista Iberoamericana de las Ciencias Biológicas y Agropecuarias

La eficiencia de reactores de lecho empacado con diferentes medios de soporte y con diversas aplicaciones ha sido ampliamente investigada. Por ejemplo, Vian, Vigueras, Velasco y Puebla (2020) evaluaron un reactor anaerobio de flujo ascendente empacado con lecho de lodos en la degradación de desechos de frutas y verduras y obtuvieron una remoción de sólidos volátiles de 67 \% en cuatro días de tiempo de retención hidráulica (TRH) con una productividad de metano $\left(\mathrm{CH}_{4}\right)$ entre 0.5 y $3.6 \mathrm{LCH}_{4} /(\mathrm{L} \cdot \mathrm{d})$ para cargas entre 1 y $10 \mathrm{~g} \mathrm{SV} /(\mathrm{L}$ - d). La productividad expresada en $\mathrm{LCH}_{4} /(\mathrm{g} \mathrm{SV}$ consumido - d) estuvo entre 0.027 y 0.116 $\mathrm{LCH}_{4} /(\mathrm{g} \mathrm{SV}$ consumido $\cdot$ d). Previamente, Vigueras, Vian, Velasco y Zafra (2016) habían diseñado un rector del mismo tipo que obtuvo una remoción de sólidos de $67 \%$ en 12 días con productividades de metano de $1.45 \mathrm{LCH}_{4} /(\mathrm{L} \cdot \mathrm{d})$, valores proporcionales a las cargas orgánicas aplicadas al sistema $(0.66-2.5 \mathrm{~g} \mathrm{SV} /(\mathrm{L} \cdot \mathrm{d})$.

Fernández et al. (2020), por su parte, estudiaron un efluente minero-metalúrgico que contenía $\mathrm{Cu}, \mathrm{Fe}$ y $\mathrm{Pb}$ en un filtro bioadsorbente con fibras lignocelulósicas (cáscaras de plátano, coco y naranja). La mejor eficiencia de remoción para el $\mathrm{Cu}$ se presentó con las cáscaras de coco $(96.36 \%)$. Para el caso del Fe, los mejores resultados se obtuvieron con mitad de cáscaras de coco y mitad de naranja (92.05\%). Y la mayor remoción de Pb se logró con una combinación de cáscaras de naranja y coco-naranja (97.34\%). En todos los tratamientos se trabajó con un pH de 7.3, con un TRH de tres horas y con un tamaño de partículas de $0.25 \mathrm{~mm}$.

Otro ejemplo más es la investigación realizada por Pérez et al. (2019), quienes evaluaron el efecto de la adición de zeolita en un reactor anaerobio de lecho granular expandido (EGSB, por sus siglas en inglés) para el tratamiento de aguas residuales porcinas con altas cargas orgánicas volumétricas. Pérez et al. (2019) encontraron que la zeolita no afectó la hidrodinámica del EGSB. Ajustado al modelo de tanques en serie, operaron dos reactores (R1 y R2) con volumen de $3.04 \mathrm{~L}$, temperatura de $30{ }^{\circ} \mathrm{C}$, un $\mathrm{TRH}$ de 12 horas y con un flujo de $4 \mathrm{~mL} / \mathrm{min}$. En la primera corrida evaluaron la estrategia de choque de carga orgánica con velocidad ascensional de $6 \mathrm{~m} / \mathrm{h}$ durante 180 días: el reactor con inclusión de zeolita alcanzó una eficiencia de $80 \%$ a $32 \mathrm{~kg}$ de demanda química de oxígeno (DQO)/m³ En la segunda corrida evaluaron un incremento gradual de carga orgánica y aumento de la velocidad ascensional hasta $10 \mathrm{~m} / \mathrm{h}$ por un periodo de 255 días: aquí se observó un incremento de eficiencia de hasta $90 \%$, la mayor velocidad de degradación orgánica. Por último, estos investigadores aseguran que adicionar $40 \mathrm{~g} / \mathrm{L}$ de zeolita a los EGSB permite 
Revista Iberoamericana de las Ciencias Biológicas y Agropecuarias

estabilizar el sistema y se vuelve robusto ante variaciones en las condiciones operacionales, con cambios favorables en la diversidad microbiana.

Asimismo, Guerrero, Vázquez y Rodríguez (2019) evaluaron la remoción de nitrógeno en un sistema tubular acondicionado con cuatro capas de zeolita de tamaños diferentes de los granos. La zeolita del tipo clinoptilolita fue adquirida comercialmente. Compraron granos de $1.70 \mathrm{~mm}, 2 \mathrm{~mm}, 2.63 \mathrm{~mm}$ y $4.75 \mathrm{~mm}$. Entre los resultados destacan que se logró remover entre $50 \%$ y $75 \%$ del nitrógeno total y especialmente el nitrógeno amoniacal. El contenido de fosfato se redujo entre $50 \%$ y $95 \%$, así como la demanda bioquímica de oxígeno, que pudo mejorarse hasta en $45 \%$. En suma, entre $2 \%$ y un $8 \%$ de los sólidos disueltos fueron eliminados, así como entre $35 \%$ y $85 \%$ de los sólidos suspendidos, y también se logró mejorar la conductividad eléctrica hasta $3 \%$ y el oxígeno disuelto entre $2 \%$ y $7 \%$.

Cárdenas y Ramos (2009) evaluaron cuatro medios de contacto (concha marina, material sintético, material vitrificado y grava de río) en un filtro anaerobio de flujo ascendente (FAFA). La concha marina alcanzó las mayores remociones (DQO de 89.7 \% y demanda de bióxido oxígeno [DBO] de $87.8 \%$ ) debido a su estructura física, que permitió un microambiente adecuado, y a su composición química, fuente natural de alcalinidad y micronutrientes. En Colombia, Rodríguez, Pinzón y Arámbula (2007) desarrollaron e implementaron un biorreactor de lecho empacado cerámico donde trataron aguas residuales domésticas. Lograron remover $78 \%$ de sólidos suspendidos volátiles, 77 \% de $\mathrm{DBO}_{5}, 57 \%$ de DQO, de $79 \%$ coliformes totales (CT) y $51 \%$ de P con un TRH de pulso y paso de 17.75 segundos y 34.577 segundos, respectivamente.

Aunado a todos los trabajos ya citados, Castillo, Solano y Rangel (2006) estudiaron en un reactor de lecho fijo de flujo descendente una biopelícula anaerobia sobre tusas de mazorca, estropajo y poliuretano sintético como material de soporte. El agua evaluada la tomaron de la Planta de Tratamiento de Aguas Residuales de Río Frío (Girón, Santander, Colombia) y otra proveniente de una laguna para el tratamiento de excretas porcícolas (Mesa de los Santos, Santander, Colombia). Castillo et al. (2006) obtuvieron para la biopelícula sobre tusas de mazorca los mayores porcentajes de remoción de DQO (50-75\%) y de composición de $\mathrm{CH}_{4}$ en el biogás (45-75\%) cuando lo alimentaron con cargas orgánicas mayores a $1500 \mathrm{mg} / \mathrm{L}$ de DQO con una temperatura de $38^{\circ} \mathrm{C}$, un TRH entre 6 y 24 horas y pH entre 6.5-7.5. Por último, Rivas, Nevárez, Bautista, Pérez y Saucedo (2003) estudiaron 
aguas residuales para ser utilizadas en la industria agrícola con un sistema biológico de lecho fijo de flujo ascendente a escala semipiloto, con variación en la altura de lecho $(\mathrm{H})$ y el diámetro del medio de soporte, manteniendo constantes el flujo de agua y aire, observaron que la eficiencia de remoción más alta de materia orgánica $(91.9 \%)$ se obtuvo cuando el biorreactor fue operado con una $\mathrm{H}$ de $0.65 \mathrm{~m}$ y un tamaño de partícula de $1.87 \mathrm{~mm}$.

A pesar de ser un tema bastante estudiado, en Tabasco, México, no hay reportes del uso de zeolitas nativas en biorreactores de lecho fijo de flujo ascendente (BLFFA). Aun cuando se cuenta con material nativo en la región, no se ha demostrado su eficacia en el tratamiento de aguas residuales. Para esta investigación se diseñó y construyó un sistema experimental de BLFFA para tratar aguas de efluentes primarios y se estudió la eficiencia de las zeolitas nativas como medio de soporte. Así, se evaluaron las características fisicoquímicas de las zeolitas y la remoción de parámetros de control como temperatura, pH, sólidos disueltos totales (SDT), color, turbiedad y DQO, para demostrar el potencial de estos materiales e incentivar su uso en las comunidades donde se implementa este tipo de procesos de tratamiento de aguas residuales. El alcance final es demostrar que las zeolitas nativas son factibles de implementar en el tratamiento secundario mediante los BLFFA en el sureste mexicano.

\section{Materiales y método}

El sistema experimental BLFFA fue construido en la División Académica de Ciencias Biológicas (DACBiol) de la Universidad Juárez Autónoma de Tabasco (UJAT). El agua residual provino de las instalaciones sanitarias de ese espacio en Villahermosa, Tabasco. Las zeolitas nativas fueron obtenidas en bancos de materiales pétreo (de cerro y de río) $\left(17^{\circ} 34^{\prime}\right.$ 27.62" N y $92^{\circ} 55^{\prime} 44.25^{\prime \prime} \mathrm{O}, 17^{\circ} 32^{\prime} 45.91^{\prime \prime} \mathrm{N}$ y $\left.92^{\circ} 55^{\prime} 56.68^{\prime \prime} \mathrm{O}\right)$ en Teapa, Tabasco. Las zeolitas se caracterizaron en el Laboratorio de Ingeniería Civil del Instituto Tecnológico de Villahermosa. La calidad del agua de los experimentos se caracterizó en el Laboratorio de Tecnología del Agua de la DACBiol. 


\section{Características del sistema experimental}

El sistema experimental BLFFA se instaló al lado del cárcamo de aguas residuales de la DACBiol-UJAT. Al agua residual utilizada en los experimentos se le aplicó previamente un tratamiento primario para eliminación de sólidos sedimentables y suspendidos mediante el sistema de tratamiento de la DACBiol, el cual consta de rejilla, desarenador, desnatador y fosa séptica. Esta agua fue transportada a través de tuberías de 1/2 pulgada por una bomba de $1 / 4$ de caballo de potencia a un tinaco con capacidad de $2.1 \mathrm{~m}^{3}$, el cual, por gravedad, alimentó al BLFFA. Las características de este se muestran en la tabla 1, figura 1 y figura 2.

Tabla 1. Características y dimensiones de cada biorreactor del sistema experimental

\begin{tabular}{|l|c|c|}
\hline \multicolumn{1}{|c|}{ Descripción } & Unidad & Valor \\
\hline Base de cada reactor y altura & $\mathrm{m}$ & 0.5 x 0.5 x 0.5 \\
\hline Diámetro del plénum & $\mathrm{m}$ & 0.1016 \\
\hline Altura del plénum & $\mathrm{m}$ & 0.20 \\
\hline Área de la base del plénum & $\mathrm{m}^{2}$ & 0.0081 \\
\hline Volumen del plénum & $\mathrm{m}^{3}$ & 0.0016 \\
\hline Diámetro de plato distribuidor con siete toberas y malla & $\mathrm{m}$ & 0.1016 \\
\hline Altura de la columna de PVC & $\mathrm{m}$ & 1.50 \\
\hline Volumen efectivo de la columna de PVC de cada reactor & $\mathrm{m}^{3}$ & 0.0122 \\
\hline
\end{tabular}

Fuente: Elaboración propia

Figura 1. Configuración del sistema experimental de BLFFA

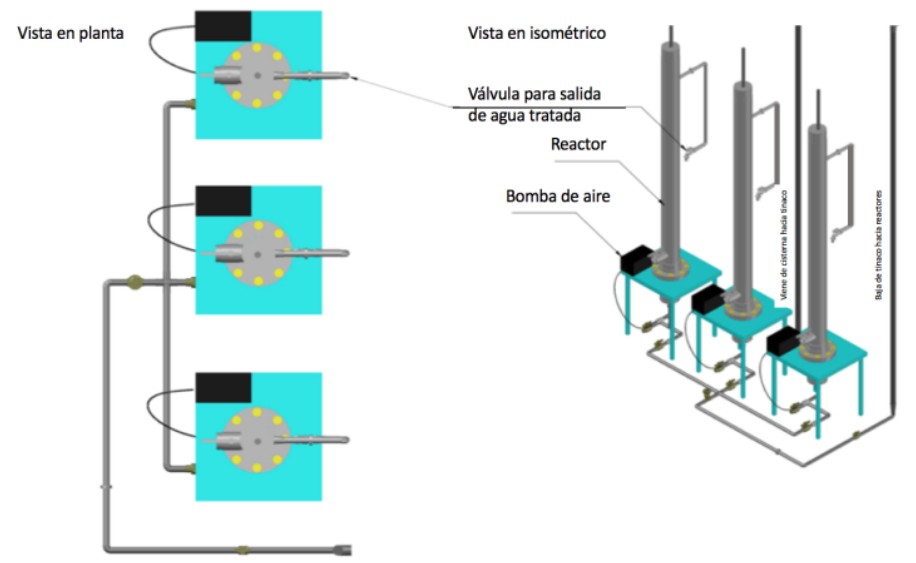

Fuente: Elaboración propia 
Revista Iberoamericana de las Ciencias Biológicas y Agropecuarias

Figura 2. Detalle interno del reactor, toberas y malla para no permitir el paso de las partículas, sobre la malla se colocó el lecho empacado de zeolitas.
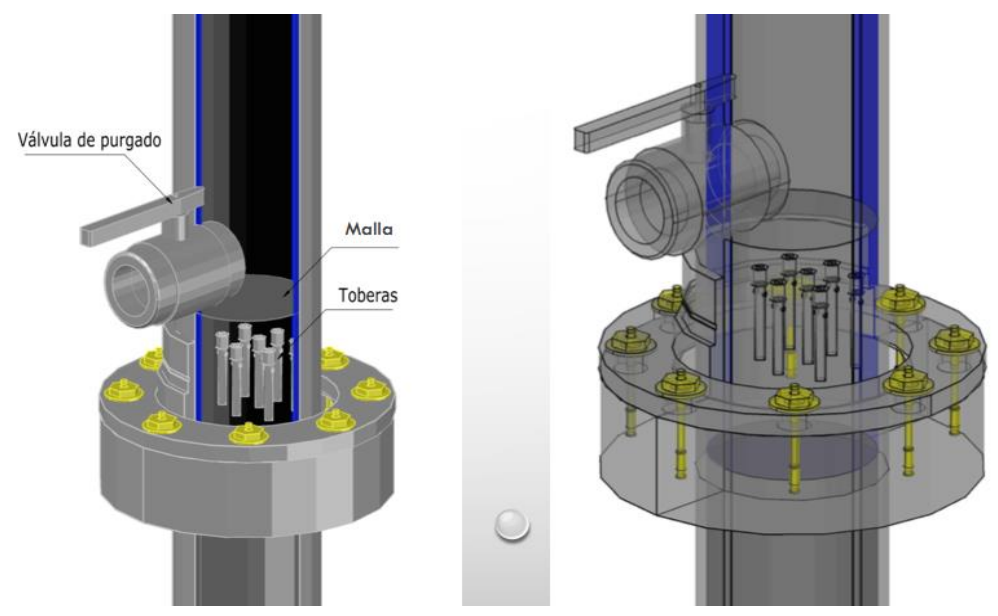

Fuente: Elaboración propia

\section{Características de las zeolitas}

Para caracterizar las zeolitas se tomaron cinco muestras de $25 \mathrm{~kg}$ de zeolita de río, zeolita de cerro (ambas trituradas de desecho en planta) y zeolita comercial (se adquirió en tienda de tratamiento de aguas). Se procesaron 10 muestras $(5 \mathrm{~kg})$ al azar de cada zeolita. La preparación incluyó el traslado, recepción, registro, secado, tamizado, homogeneizado y el almacenamiento para su conservación, evitando la contaminación de la muestra y asegurando precisión y exactitud en el análisis. A las muestras se les determinó el pH, la conductividad eléctrica (CE), densidad aparente y real, peso específico, porosidad y solubilidad en ácido clorhídrico, tal y como lo establece Muñoz, Soler, López y Hernández (2015) y la NOM021-RECNAT-2000.

\section{Monitoreo del sistema experimental}

El muestreo se realizó bajo la NOM-AA-3-1980 después de tres meses de estabilización (febrero-abril del 2018). En la fase experimental con los BLFFA (mayooctubre 2018), se midieron los parámetros de control lunes, miércoles y viernes, tomando tres muestras simples obtenidas cada seis horas (6:00 a. m., 12:00 m. y 6:00 p. m.). Los parámetros determinados fueron temperatura, $\mathrm{pH}$, SDT, color, turbiedad y DQO. Estos se midieron al inicio y final de cada experimento, tomando la muestra de agua residual (influente) del cárcamo y la muestra de salida (efluente) después de cada experimento. Los 
Revista Iberoamericana de las Ciencias Biológicas y Agropecuarias

métodos usados para la medición de los parámetros fueron tomados de Standard Methods for the Examination of Water and Wastewater (2017): 2550 Temperature (2017), 2540 Total Dissolved Solids y 4500-H+ pH Value , medidos con el equipo Hanna HI98129. La turbiedad se determinó por el método EPA 180.1, TC-300e, ISO 7027, TC-300i, utilizando un equipo Hanna HI 98703. El color se determinó mediante el método 2120 Color (Standard Methods for the Examination of Water and Wastewater, 2017), con un equipo Lamotte. El oxígeno disuelto (OD) se determinó por el 4500-H+ pH Value (Standard Methods for the Examination of Water and Wastewater, 2017). La DQO por el método EPA 410.4 (O'Dell, 1993). Cada experimento se realizó por triplicado, esto es, un total de 27 experimentos y 81 muestras de los experimentos. El sistema experimental BLFFA fue operado a temperatura ambiente, con un suministro de aire que permitiera mantener en reactor $4.5 \pm 0.7 \mathrm{mg} / \mathrm{L}$ de OD respectivamente en los tratamientos.

\section{Diseño experimental}

En la corrida experimental se empleó un diseño completamente al azar en un arreglo factorial de $3 \times 3$, con tres repeticiones para el tratamiento. Se utilizaron tres BLFFA. Cada corrida duró dos meses (Rivas et al., 2003) y los factores fueron los tres tipos zeolita con $\varnothing$ de partícula de $1 / 4$ pulgada de forma irregular, la altura del lecho $(0.75 \mathrm{~m}, 0.90 \mathrm{~m}, 1.10 \mathrm{~m})$ y el TRH (6, 12, 24 horas) con caudales de 48.6, 24.3, 12.2 L/día respectivamente en cada reactor.

\section{Análisis estadístico y eficiencia en el sistema experimental}

Todos los resultados obtenidos fueron evaluados en el programa estadístico Statgraphics Centurion XVI.II. Se compararon las variables de respuestas cuantitativas temperatura, $\mathrm{pH}$, SDT, turbiedad, color y DQO de los diferentes tipos de tratamientos de las zeolitas, las alturas de lecho y los TRH. Para determinar si existían diferencias estadísticas significativas, se realizó un análisis de varianza (Anova) multifactorial y el método empleado para discriminar entre las medias fue el procedimiento de diferencia mínima significativa (LSD, por sus siglas en inglés) de Fisher con una $p<0.05$ y con $95 \%$ de nivel de confianza. El desempeño del biorreactor fue evaluado con base en los resultados de las diferencias de concentración entre el afluente y el efluente utilizando los datos promedios de los contaminantes, como lo establece Torres, López, Cerino, Vázquez y Comparán (2020). 


\section{Resultados \\ Características de las zeolitas}

En la tabla 2 se presentan los resultados de la caracterización fisicoquímica realizada a las zeolitas. Estas presentan densidad real y aparente muy similar entre las tres. Sin embargo, destaca que la zeolita de cerro presenta mayores atributos en porosidad, característica muy importante en el soporte de biomasa bacteriana; también presentó la menor solubilidad en medio ácido clorhídrico.

Tabla 2. Características de las zeolitas. Valores promedio $(N=10)$

\begin{tabular}{|l|c|c|c|}
\hline \multicolumn{1}{|c|}{ Parámetro } & Zeolita de río & Zeolita de cerro & Zeolita comercial \\
\hline Densidad real $\left(\mathrm{kg} / \mathrm{m}^{3}\right)$ & 2600 & 2700 & 2650 \\
\hline Densidad aparente $\left(\mathrm{kg} / \mathrm{m}^{3}\right)$ & 1550 & 1470 & 1600 \\
\hline Peso específico $\left(\mathrm{kg} / \mathrm{m}^{3}\right)$ & 2290 & 2420 & 2350 \\
\hline Absorción (\%) & 14.10 & 15.20 & 15.00 \\
\hline Porosidad (\%) & 53.00 & 62.00 & 51.00 \\
\hline CE (mS/cm) & 65.00 & 70.00 & 67.00 \\
\hline pH (UpH) & 7.30 & 7.40 & 7.00 \\
\hline $\begin{array}{l}\text { Solubilidad en ácido } \\
\text { clorhídrico (\%) }\end{array}$ & 59.66 & 29.96 & 51.30 \\
\hline
\end{tabular}

Fuente: Elaboración propia

\section{Evaluación de parámetros de control y contaminantes básicos}

El agua residual con las que se desarrolló el experimento presentó (promedio \pm DE, $N=9$ ) una temperatura $25.26 \pm 1.76{ }^{\circ} \mathrm{C}$, $\mathrm{pH}$ de $8.63 \pm 0.23$, SDT con $950.50 \pm 115.05 \mathrm{mg} / \mathrm{L}$, color de $1305.10 \pm 141.46$ UC, turbiedad 96.37 \pm 7.79 UNT y DQO $373 \pm 43 \mathrm{mg} / \mathrm{L}$. Esta agua residual es de concentración media, pues la DQO está en el rango media-débil (250-500 mg/L), según Tchobanoglous, Burton y Stensel (2003). En la tabla 3 se presentan los resultados de los efluentes en los diferentes tratamientos de los BLFFA. Allí se observa que el tratamiento con zeolita de río presentó la menor concentración de DQO en la H de 0.90 m y TRH de 24 horas con $23.46 \mathrm{mg} / \mathrm{L}$; en cuanto a la zeolita de cerro, la menor concentración se dio en la $\mathrm{H}$ de $0.90 \mathrm{~m}$ y TRH de 24 horas con $9.86 \mathrm{mg} / \mathrm{L}$, y con la zeolita comercial la 
Revista Iberoamericana de las Ciencias Biológicas

y Agropecuarias

menor concentración se suscitó con el tratamiento H de 0.90 m y TRH de 12 horas con 11.87 $\mathrm{mg} / \mathrm{L}$.

Tabla 3. Contaminantes básicos del agua residual en el efluente de los BLFFA en cada tratamiento. Valores promedio y $\mathrm{DE}(N=81)$

\begin{tabular}{|c|c|c|c|c|c|c|c|c|c|c|}
\hline & Parámetro & & $=0.75$ & & & $=0.90$ & & & $=1.10$ & \\
\hline & & $6 \mathrm{~h}$ & $12 \mathrm{~h}$ & $24 \mathrm{~h}$ & $6 \mathrm{~h}$ & $12 \mathrm{~h}$ & $24 \mathrm{~h}$ & $6 \mathrm{~h}$ & $12 \mathrm{~h}$ & $24 \mathrm{~h}$ \\
\hline & $\begin{array}{l}\text { Temp. } \\
\left({ }^{\circ} \mathrm{C}\right)\end{array}$ & 28.93 & 29.77 & 28.83 & 28.87 & 29.17 & 28.77 & 29.53 & 30.07 & 29.53 \\
\hline & $\mathrm{pH}(\mathrm{UpH})$ & 9.67 & 8.80 & 8.73 & 8.00 & 7.50 & 7.27 & 7.93 & 7.47 & 7.23 \\
\hline$\stackrel{0}{\varrho}$ & SDT & 993.0 & 902.3 & 889.4 & 830.7 & 817.7 & 805.2 & 827.4 & 820.4 & 805.4 \\
\hline$\underset{\pi}{\pi}$ & $(\mathrm{mg} / \mathrm{L})$ & 7 & 0 & 0 & 7 & 7 & 0 & 7 & 0 & 3 \\
\hline 8 & Color & 824.0 & 777.4 & 728.3 & 489.1 & 235.1 & 234.1 & 478.5 & 237.2 & 236.2 \\
\hline & (UC) & 5 & 2 & 4 & 3 & 3 & 0 & 3 & 7 & 7 \\
\hline & $\begin{array}{l}\text { Turb. } \\
\text { (UNT) }\end{array}$ & 16.82 & 15.87 & 14.86 & 39.27 & 20.87 & 8.17 & 38.67 & 19.83 & 7.97 \\
\hline & $\begin{array}{l}\text { DQO } \\
(\mathrm{mg} / \mathrm{L})\end{array}$ & 48.31 & 45.58 & 42.70 & $\begin{array}{c}112.8 \\
1\end{array}$ & 59.95 & 23.46 & $\begin{array}{c}111.0 \\
8\end{array}$ & 56.98 & 22.89 \\
\hline & Parámetro & & $=0.75$ & & & $=0.90$ & & & $=1.10$ & \\
\hline & & $6 \mathrm{~h}$ & $12 \mathrm{~h}$ & $24 \mathrm{~h}$ & $6 \mathrm{~h}$ & $12 \mathrm{~h}$ & $24 \mathrm{~h}$ & $6 \mathrm{~h}$ & $12 \mathrm{~h}$ & $24 \mathrm{~h}$ \\
\hline & $\begin{array}{l}\text { Temp. } \\
\left({ }^{\circ} \mathrm{C}\right)\end{array}$ & 29.83 & 30.00 & 30.40 & 29.17 & 28.77 & 29.17 & 30.07 & 29.53 & 30.07 \\
\hline & $\mathrm{pH}(\mathrm{UpH})$ & 9.40 & 8.50 & 8.47 & 7.97 & 6.97 & 6.90 & 7.87 & 6.97 & 6.90 \\
\hline$\overline{0}$ & SDT & 965.8 & 873.5 & 873.0 & 591.1 & 271.6 & 266.6 & 528.0 & 271.1 & 266.7 \\
\hline 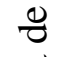 & $(\mathrm{mg} / \mathrm{L})$ & 0 & 3 & 0 & 3 & 7 & 3 & 0 & 3 & 0 \\
\hline$=$ & Color & 820.7 & 768.3 & 768.4 & 470.8 & 204.4 & 203.4 & 470.8 & 208.9 & 206.9 \\
\hline$N$ & (UC) & 5 & 9 & 2 & 0 & 3 & 3 & 0 & 7 & 7 \\
\hline & $\begin{array}{l}\text { Turb. } \\
\text { (UNT) }\end{array}$ & 16.75 & 15.68 & 15.68 & 20.80 & 3.53 & 3.43 & 20.53 & 3.67 & 3.60 \\
\hline & $\begin{array}{l}\text { DQO } \\
(\mathrm{mg} / \mathrm{L})\end{array}$ & 48.12 & 45.05 & 45.05 & 59.75 & 10.15 & 9.86 & 58.99 & 10.53 & 10.34 \\
\hline
\end{tabular}


Revista lberoamericana de las Ciencias Biológicas y Agropecuarias

\begin{tabular}{|c|c|c|c|c|c|c|c|c|c|c|}
\hline \multirow{10}{*}{ 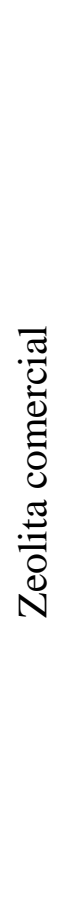 } & \multirow[t]{2}{*}{ Parámetro } & \multicolumn{3}{|c|}{$\mathrm{H}=0.75 \mathrm{~m}$} & \multicolumn{3}{|c|}{$\mathrm{H}=0.90 \mathrm{~m}$} & \multicolumn{3}{|c|}{$\mathrm{H}=1.10 \mathrm{~m}$} \\
\hline & & $6 \mathrm{~h}$ & $12 \mathrm{~h}$ & $24 \mathrm{~h}$ & $6 \mathrm{~h}$ & $12 \mathrm{~h}$ & $24 \mathrm{~h}$ & $6 \mathrm{~h}$ & $12 \mathrm{~h}$ & $24 \mathrm{~h}$ \\
\hline & $\begin{array}{l}\text { Temp. } \\
\left({ }^{\circ} \mathrm{C}\right)\end{array}$ & 30.07 & 30.87 & 31.53 & 28.77 & 29.17 & 28.77 & 29.53 & 30.07 & 29.53 \\
\hline & $\mathrm{pH}(\mathrm{UpH})$ & 9.37 & 8.53 & 8.43 & 7.93 & 6.83 & 6.77 & 7.97 & 6.80 & 6.83 \\
\hline & SDT & 965.5 & 874.3 & 873.2 & 731.4 & 698.7 & 424.4 & 732.6 & 681.4 & 412.1 \\
\hline & $(\mathrm{mg} / \mathrm{L})$ & 3 & 0 & 0 & 7 & 0 & 0 & 0 & 0 & 3 \\
\hline & Color & 820.6 & 768.3 & 768.5 & 476.0 & 206.9 & 205.9 & 475.1 & 206.8 & 205.8 \\
\hline & (UC) & 8 & 7 & 3 & 0 & 0 & 0 & 0 & 3 & 3 \\
\hline & $\begin{array}{l}\text { Turb. } \\
\text { (UNT) }\end{array}$ & 16.75 & 15.68 & 15.68 & 20.97 & 4.13 & 4.33 & 20.63 & 4.27 & 4.23 \\
\hline & $\begin{array}{l}\text { DQO } \\
(\mathrm{mg} / \mathrm{L})\end{array}$ & 48.12 & 45.05 & 45.06 & 60.23 & 11.87 & 12.45 & 59.28 & 12.26 & 12.16 \\
\hline
\end{tabular}

Fuente: Elaboración propia

\section{Temperatura}

El análisis Anova multifactorial ( $p<0.05,95 \%$ de nivel confianza) mostró que el factor Zeolita tiene un efecto estadísticamente significativo sobre Temperatura. La menor temperatura se mostró en la zeolita de río $\left(29.27{ }^{\circ} \mathrm{C}\right)$, seguido de la zeolita de cerro (29.66 ${ }^{\circ} \mathrm{C}$ ) y finalmente la zeolita comercial $\left(29.81{ }^{\circ} \mathrm{C}\right.$ ) (figura 3). El factor Altura tiene un efecto estadísticamente significativo sobre Temperatura. La temperatura más baja se mostró con la $\mathrm{H}$ de $0.9 \mathrm{~m}\left(28.95{ }^{\circ} \mathrm{C}\right)$, seguido de la $\mathrm{H}$ de $1.1 \mathrm{~m}\left(29.77^{\circ} \mathrm{C}\right)$ y finalmente con la $\mathrm{H}$ de $0.75 \mathrm{~m}$ $\left(30.02{ }^{\circ} \mathrm{C}\right.$ ) (figura 4). Para el factor TRH ninguno de los factores tuvo un efecto estadísticamente significativo sobre Temperatura. La menor temperatura se registró a seis horas de TRH $\left(29.62{ }^{\circ} \mathrm{C}\right)$ (figura 5). 
Figura 3. Valores de temperatura en cada Zeolita. Contraste de medias (medias $\pm \mathrm{EE}$ )

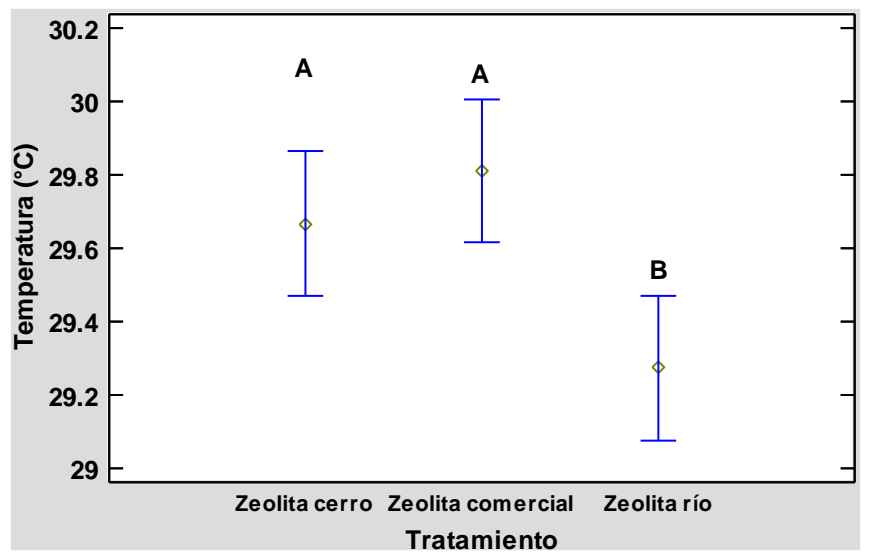

Nota: Letras distintas muestran diferencias estadísticamente significativas $(N=18)$.

Fuente: Elaboración propia

Figura 4. Valores de temperatura en cada $\mathrm{H}$. Contraste de medias (medias $\pm \mathrm{EE}$ )

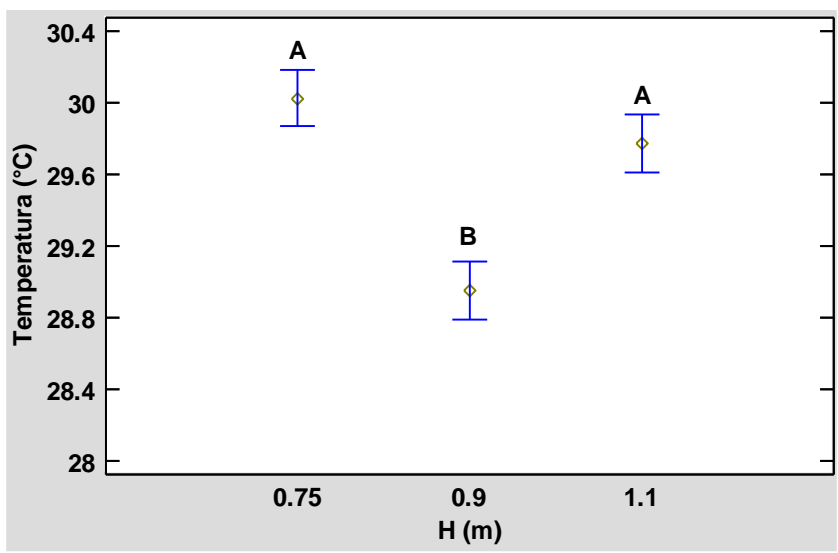

Nota: Letras distintas muestran diferencias estadísticamente significativas $(N=18)$.

Fuente: Elaboración propia 
Figura 5. Valores de temperatura en cada TRH. Contraste de medias (medias \pm EE)

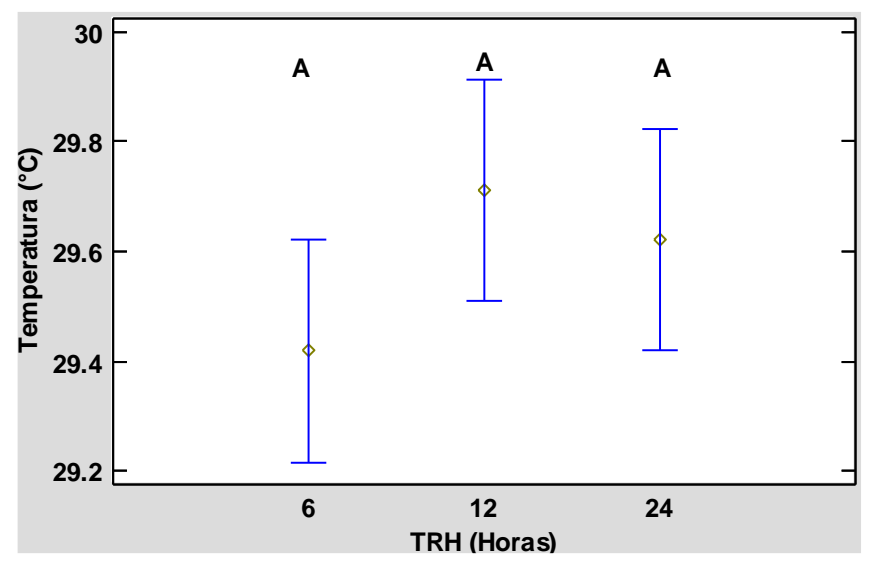

Nota: Letras distintas muestran diferencias estadísticamente significativas $(N=18)$.

Fuente: Elaboración propia

\section{pH}

El análisis Anova multifactorial ( $p<0.05,95 \%$ de nivel confianza) mostró que dos valores tienen un efecto estadísticamente significativo sobre $\mathrm{pH}$. La zeolita de río fue la que presentó un pH con tendencia alcalina (8.07) y los tratamientos de zeolita de cerro y zeolita comercial tendieron a comportarse más estables (7.77 y 7.71) (figura 6). El factor Altura tuvo un comportamiento bastante homogéneo para el $\mathrm{pH}$ en $0.9 \mathrm{~m}$ y $1.1 \mathrm{~m}(7.35$ y 7.33$)$ y el pH en $0.75 \mathrm{~m}$ presentó tendencia alcalina (8.88) (figura 7). Finalmente, en el tratamiento de seis horas se presentó un $\mathrm{pH}$ alcalino (8.45) y para los otros tiempos el $\mathrm{pH}$ tiende a la neutralidad (figura 8).

Figura 6. Valores de $\mathrm{pH}$ en cada zeolita. Contraste de medias (medias $\pm \mathrm{EE}$ )

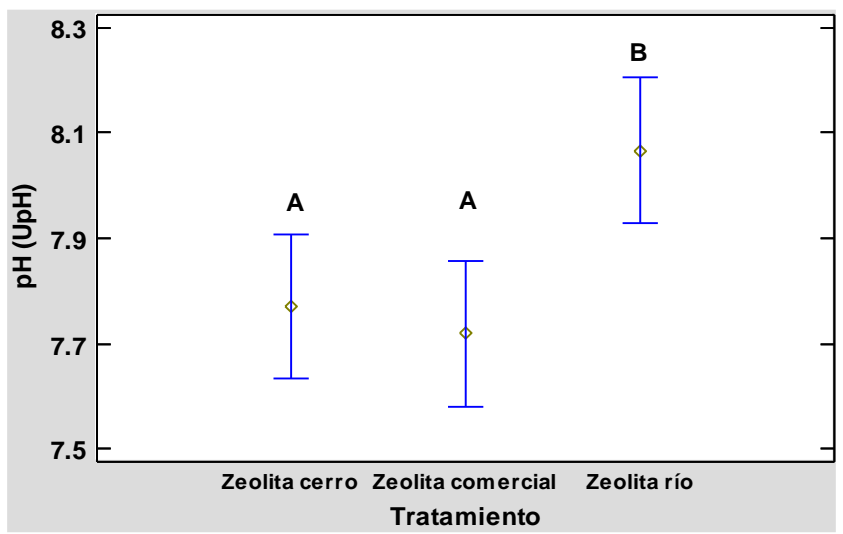

Nota: Letras distintas muestran diferencias estadísticamente significativas $(N=18)$.

Fuente: Elaboración propia 
Figura 7. Valores de $\mathrm{pH}$ en cada $\mathrm{H}$. Contraste de medias (medias $\pm \mathrm{EE}$ )

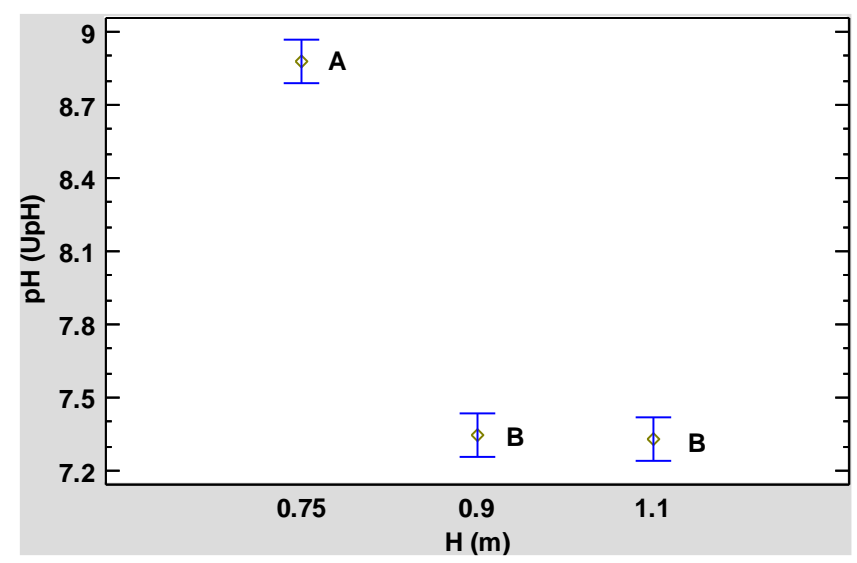

Nota: Letras distintas muestran diferencias estadísticamente significativas $(N=18)$.

Fuente: Elaboración propia

Figura 8. Valores de $\mathrm{pH}$ en cada TRH. Contraste de medias (medias $\pm \mathrm{EE}$ )

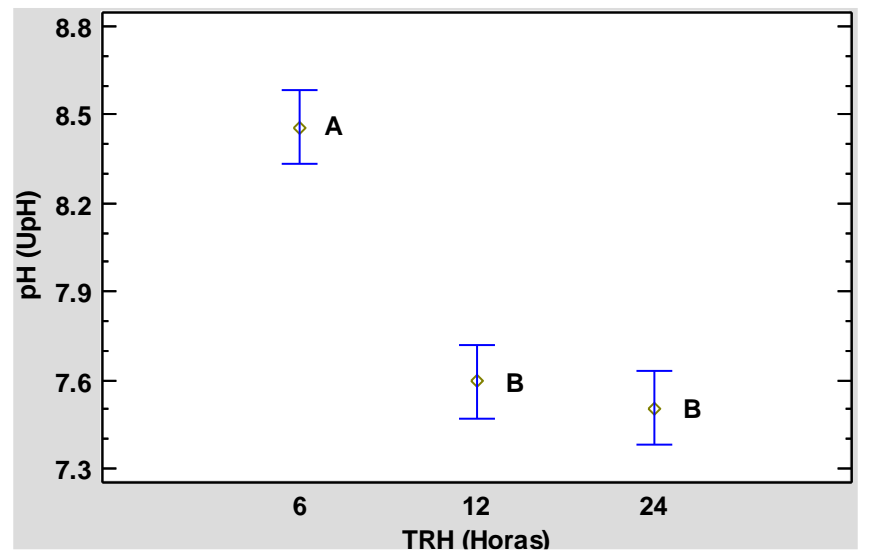

Nota: Letras distintas muestran diferencias estadísticamente significativas $(N=18)$.

Fuente: Elaboración propia

\section{Sólidos disueltos totales}

El análisis Anova multifactorial ( $p<0.05,95 \%$ de nivel confianza) muestra que el factor Zeolita tiene un efecto estadísticamente significativo sobre SDT. Aquí la zeolita de cerro fue la que presentó la menor media (545.28 mg/L), seguido de la de río (854.64 mg/L) y finalmente la zeolita comercial (1000.55 mg/L) (figura 9). El factor Altura presenta un efecto significativo en los SDT. La menor concentración fue para la H de $1.1 \mathrm{~m}$ (593.91 mg/L), seguido de la H de 0.9 m (604.19 mg/L) y finalmente la H de 0.75 (1202.37 mg/L) (figura 10). Para el factor TRH, la menor concentración se presentó a las 12 horas (690.13 
mg/L), seguido de las seis horas (796.20 mg/L) y la más alta a las 24 horas $(914.14 \mathrm{mg} / \mathrm{L})$ (figura 11).

Figura 9. Valores de SDT en cada zeolita. Contraste de medias (medias \pm EE)

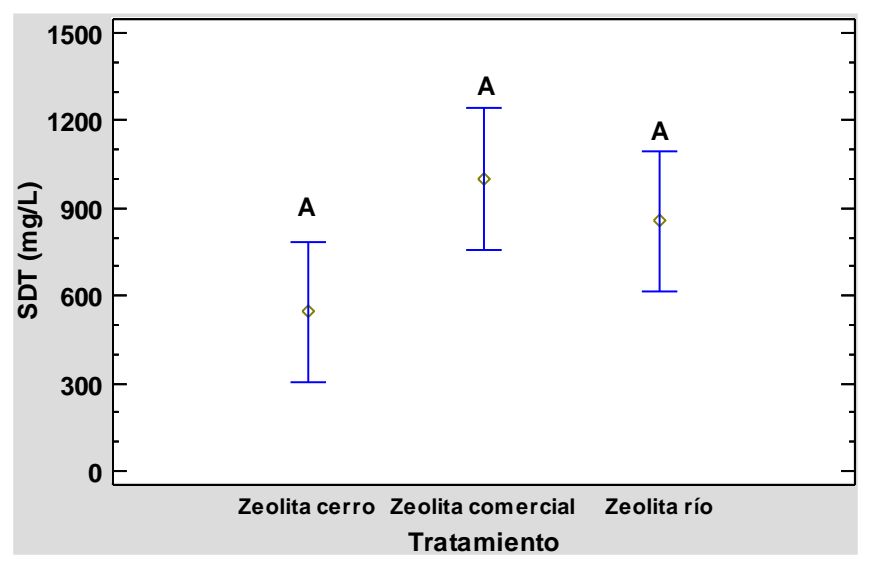

Nota: Letras distintas muestran diferencias estadísticamente significativas $(N=18)$.

Fuente: Elaboración propia

Figura 10. Valores de SDT en cada H. Contraste de medias (medias \pm EE)

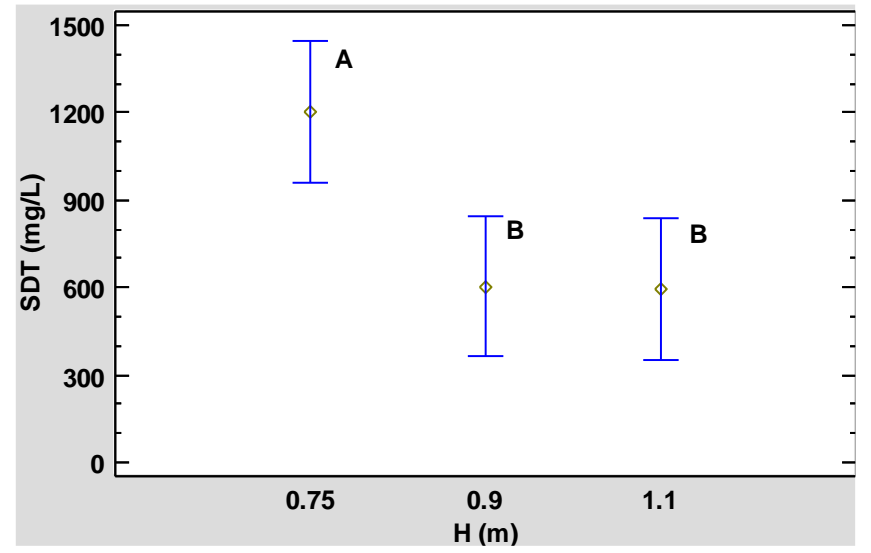

Nota: Letras distintas muestran diferencias estadísticamente significativas $(N=18)$.

Fuente: Elaboración propia 
Figura 11. Valores de SDT en cada TRH. Contraste de medias (medias $\pm \mathrm{EE}$ )

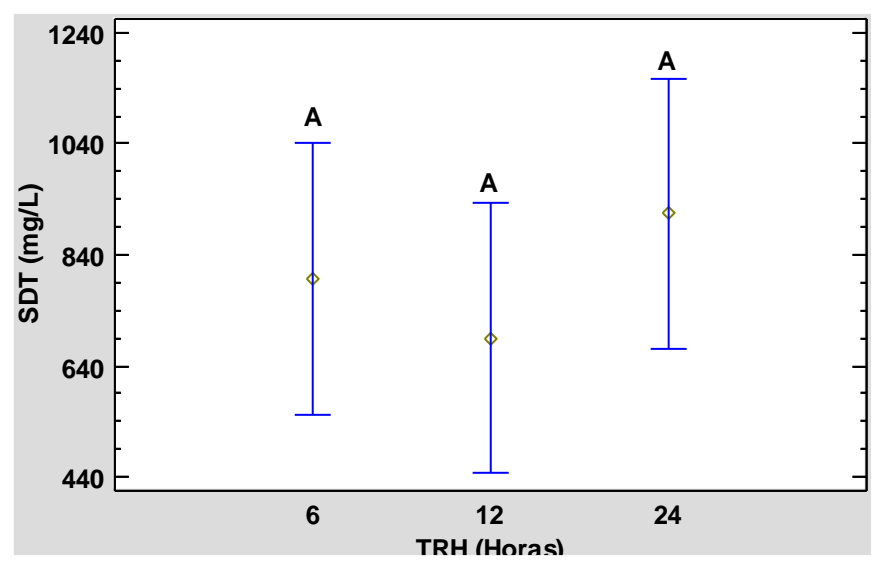

Nota: Letras distintas muestran diferencias estadísticamente significativas $(N=18)$.

Fuente: Elaboración propia

\section{Color}

El análisis Anova multifactorial ( $p<0.05,95 \%$ de nivel confianza) muestra que el factor Zeolita no tiene un efecto estadísticamente significativo sobre el color. La zeolita de cerro fue la que presentó la menor media (458.10 UC), seguido de la comercial (459.35 UC) y finalmente la zeolita de río (471.14 UC) (figura 12). El factor Altura presenta un efecto significativo en el color. La menor concentración fue para la H de 0.9 m (302.87 UC), seguido de la $\mathrm{H}$ de $1.1 \mathrm{~m}$ (302.95 UC) y finalmente la $\mathrm{H}$ de 0.75 (782.77 UC) (figura 13). Para el factor TRH la menor concentración se presentó a las 24 horas (395.30 UC), seguido de las 12 horas (401.52 UC) y la más alta a las seis horas (591.76 UC) (figura 14). 
Figura 12. Valores de color en cada Zeolita. Contraste de medias (medias \pm EE)

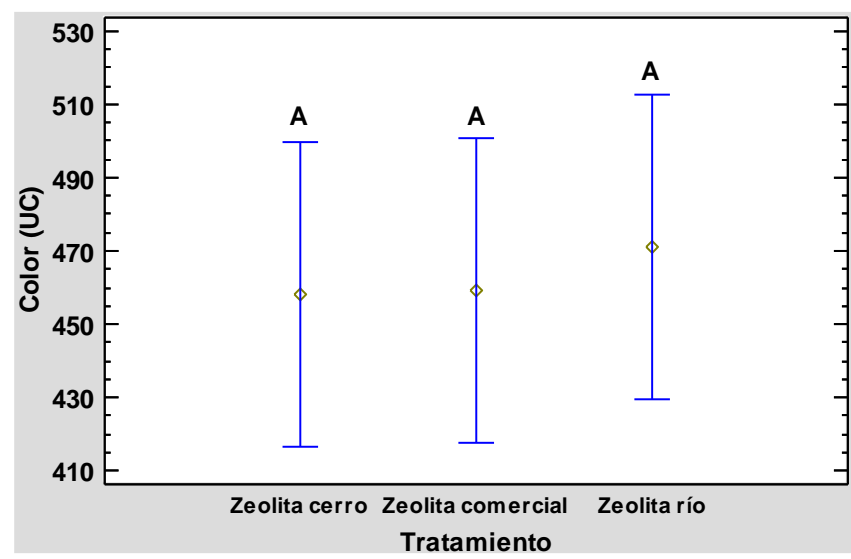

Nota: Letras distintas muestran diferencias estadísticamente significativas $(N=18)$.

Fuente: Elaboración propia

Figura 13. Valores de color en cada $\mathrm{H}$. Contraste de medias (medias $\pm \mathrm{EE}$ )

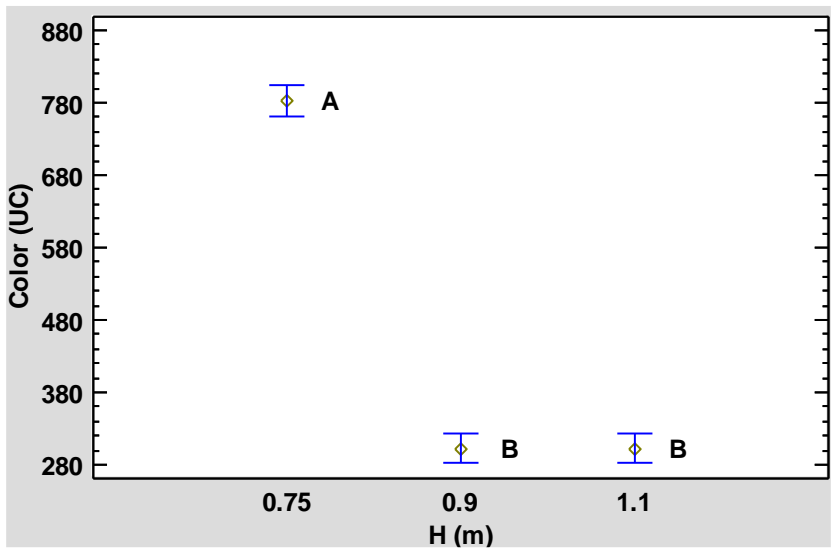

Nota: Letras distintas muestran diferencias estadísticamente significativas $(N=18)$.

Fuente: Elaboración propia 
Figura 14. Valores de color en cada TRH. Contraste de medias (medias \pm EE)

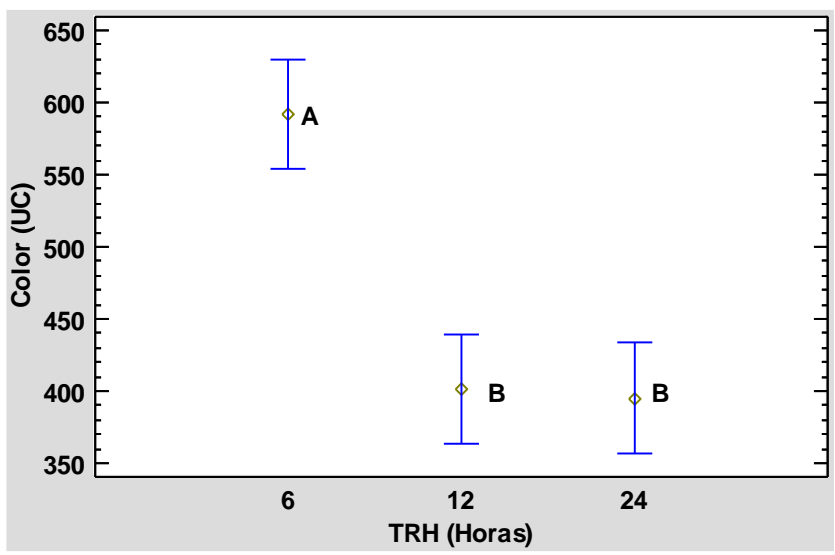

Nota: Letras distintas muestran diferencias estadísticamente significativas $(N=18)$.

Fuente: Elaboración propia

\section{Turbiedad}

El análisis Anova multifactorial ( $p<0.05,95 \%$ de nivel confianza) muestra que el factor Zeolita tiene un efecto estadísticamente significativo sobre la turbiedad. La zeolita de cerro presentó la menor media (11.52 UNT), seguido de la comercial (11.85 UNT) y finalmente la zeolita de río (20.26 UNT) (figura 15). El factor Altura presenta un efecto significativo en la turbiedad. La menor concentración se presentó en la H de $1.1 \mathrm{~m}$ (13.71 UNT), seguido de la H de 0.9 m (13.94 UNT) y finalmente la H de 0.75 (15.98 UNT) (figura 16). Para el factor TRH, la menor concentración se presentó a las 24 horas (8.67 UNT), seguido de las 12 horas (11.51 UNT) y la más alta a las seis horas (23.46 UNT) (figura 17). 
Figura 15. Valores de turbiedad en cada zeolita. Contraste de medias (medias \pm EE)

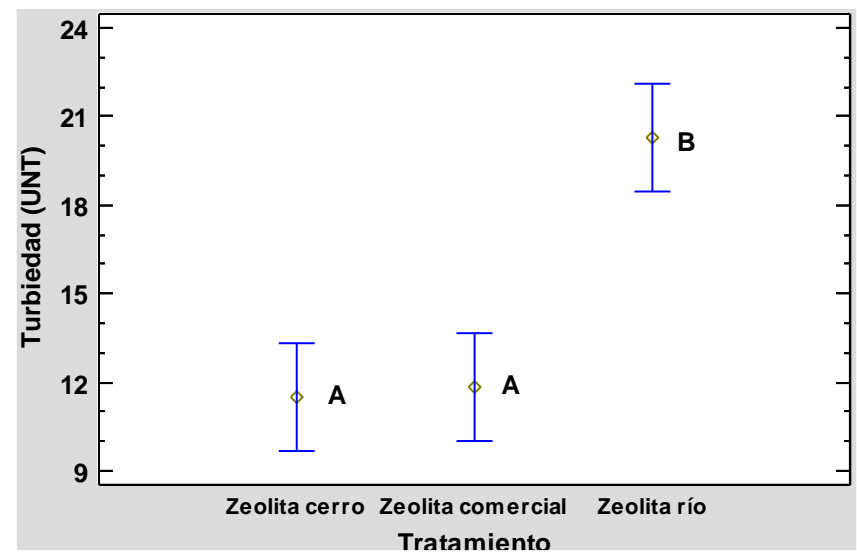

Nota: Letras distintas muestran diferencias estadísticamente significativas $(N=18)$.

Fuente: Elaboración propia

Figura 16. Valores de turbiedad en cada $\mathrm{H}$. Contraste de medias (medias $\pm \mathrm{EE}$ )

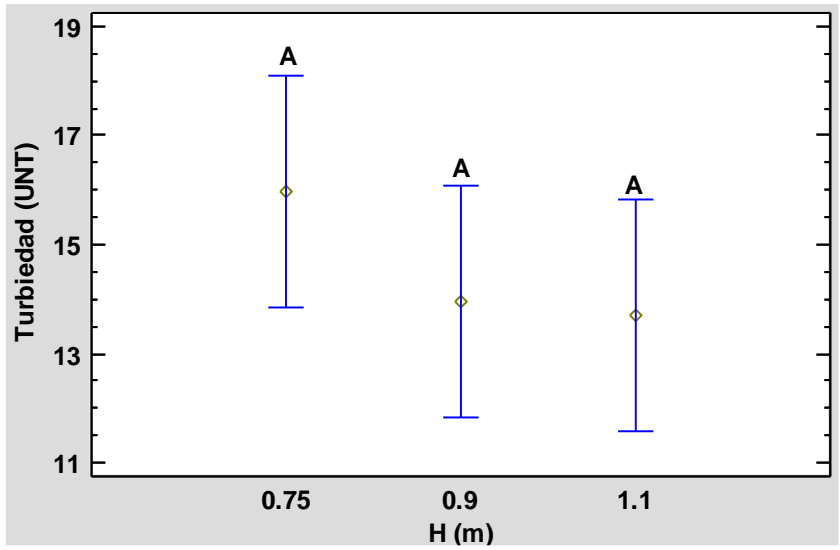

Nota: Letras distintas muestran diferencias estadísticamente significativas $(N=18)$.

Fuente: Elaboración propia 
Figura 17. Valores de turbiedad en cada TRH. Contraste de medias (medias \pm EE)

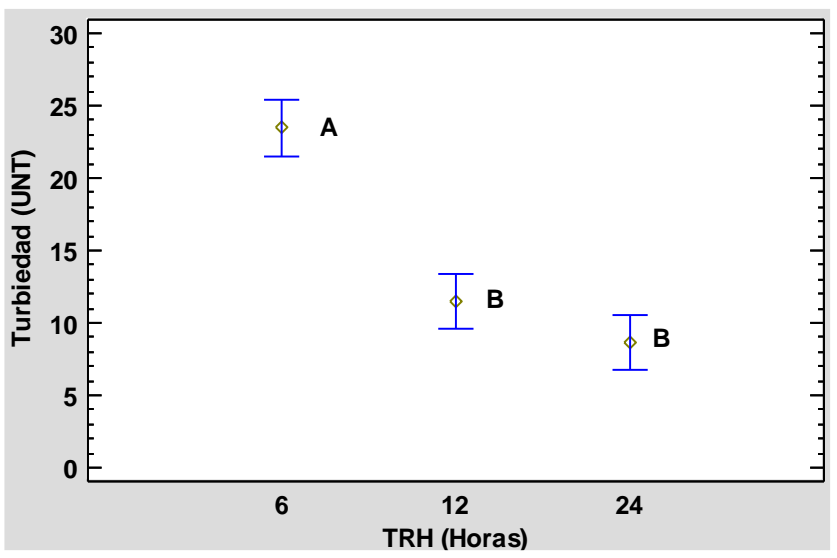

Nota: Letras distintas muestran diferencias estadísticamente significativas $(N=18)$.

Fuente: Elaboración propia

\section{Demanda química de oxígeno}

El análisis Anova multifactorial $(p<0.05,95 \%$ de nivel confianza) muestra que el factor Zeolita tiene un efecto estadísticamente significativo sobre la DQO. La zeolita de cerro fue la que presentó la menor media $(33.09 \mathrm{mg} / \mathrm{L})$, seguido de la comercial (34.05 mg/L) y finalmente la zeolita de río (58.20 mg/L) (Figura 18). El factor Altura no presenta un efecto significativo en la DQO. La menor concentración se dio en la H de $1.1 \mathrm{~m}(39.40 \mathrm{mg} / \mathrm{L})$, seguido de la $\mathrm{H}$ de $0.9 \mathrm{~m}$ (40.07 mg/L) y finalmente la $\mathrm{H}$ de 0.75 (45.88 mg/L) (figura 19). Para el factor TRH si hay un efecto significativo y la menor concentración se presentó a las 24 horas $(24.90 \mathrm{mg} / \mathrm{L})$, seguido de las 12 horas $(33.05, \mathrm{mg} / \mathrm{L})$ y la más alta a las 6 horas (67.40 mg/L) (Figura 20). 
Figura 18. Valores de DQO en cada Zeolita. Contraste de medias (medias \pm EE)

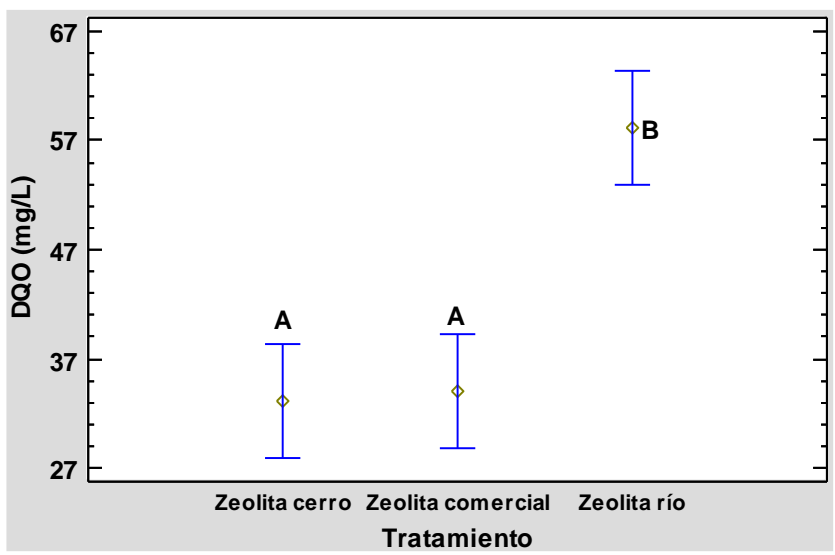

Nota: Letras distintas muestran diferencias estadísticamente significativas $(N=18)$.

Fuente: Elaboración propia

Figura 19. Valores de DQO en cada H. Contraste de medias (medias \pm EE)

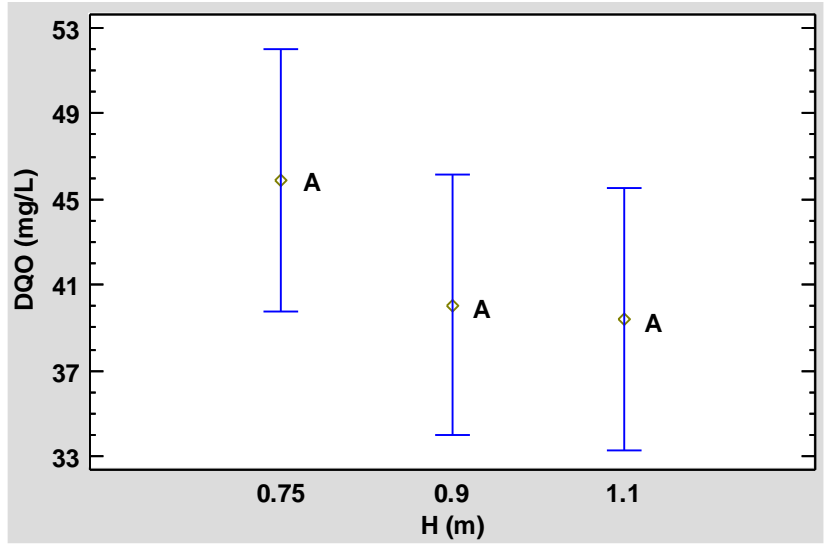

Nota: Letras distintas muestran diferencias estadísticamente significativas $(N=18)$.

Fuente: Elaboración propia 
Figura 20. Valores de DQO en cada TRH. Contraste de medias (medias \pm EE)

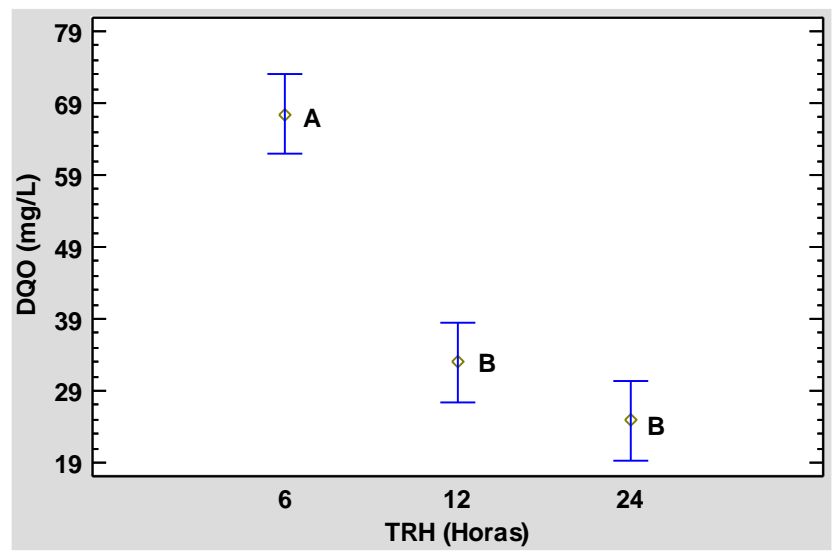

Nota: Letras distintas muestran diferencias estadísticamente significativas $(N=18)$. Fuente: Elaboración propia

\section{Eficiencia de remoción de contaminantes básicos}

En la tabla 4 se describen las eficiencias de remoción de los parámetros evaluados en los tratamientos. En la zeolita de río y de cerro el tratamiento más óptimo se presentó con una $\mathrm{H}$ de 0.9 y con 24 horas de TRH; para la zeolita comercial, con el tratamiento de $\mathrm{H}$ de $0.9 \mathrm{~m}$ con 12 horas. Esto nos permite observar que el mejor tratamiento es de zeolita de cerro, pues alcanzó la mayor eficiencia con $71.95 \%$ para SDT, $84.41 \%$ para color, $96.44 \%$ para turbiedad y $97.36 \%$ para DQO. Es importante aclarar que el signo negativo (-) en la eficiencia de remoción nos indica que el valor del parámetro es más alto en la salida y es menor en la entrada, importante para el caso de la temperatura, que nos muestra el incremento de este valor en el efluente como consecuencia de la actividad microbiana en el BLFFA. 
Revista lberoamericana de las Ciencias Biológicas

y Agropecuarias

Tabla 4. Eficiencias de remoción de contaminantes básicos en los BLFFA para diferentes tratamientos (zeolita, H y TRH) $(N=81)$

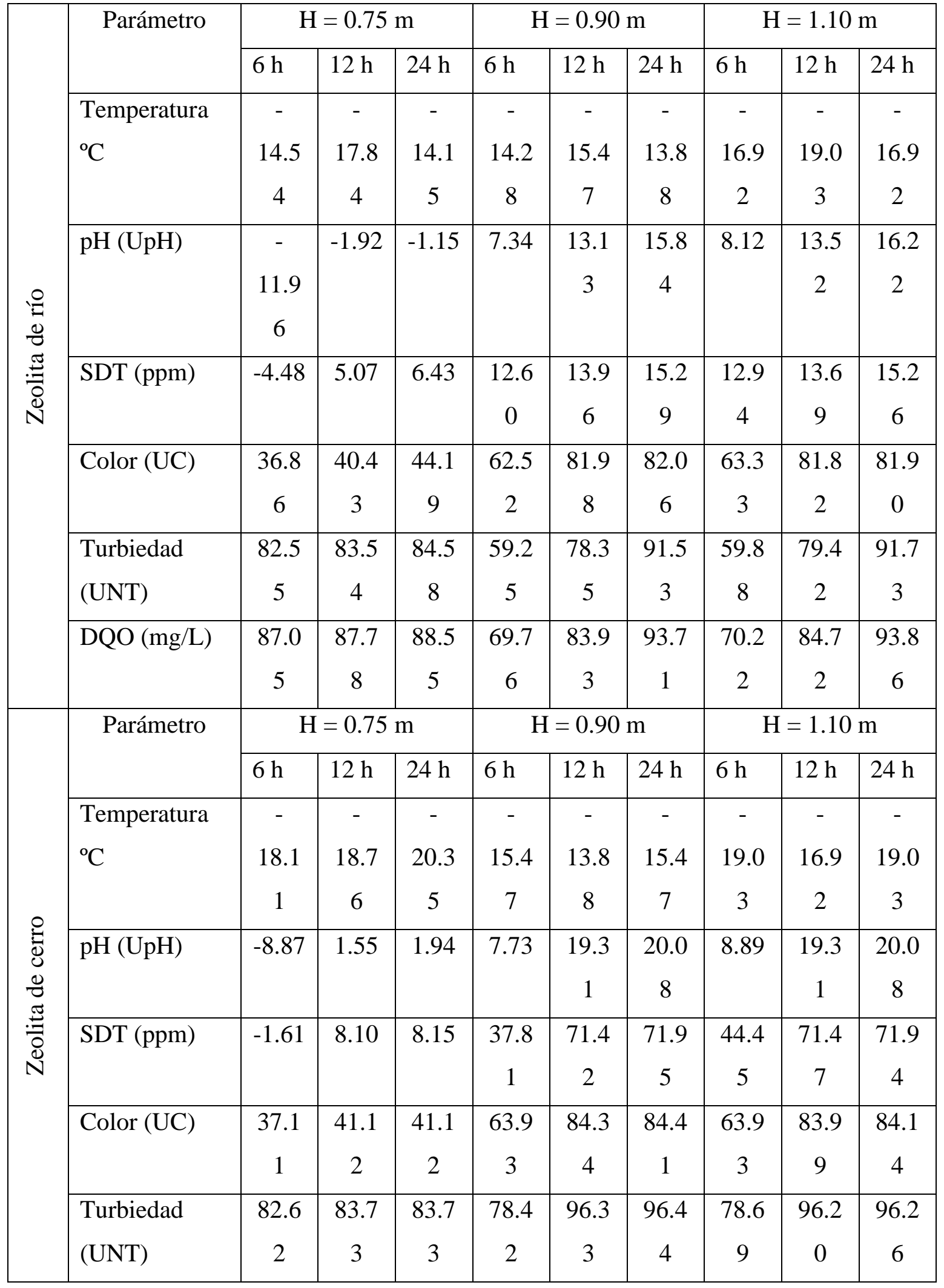


Revista lberoamericana de las Ciencias Biológicas y Agropecuarias

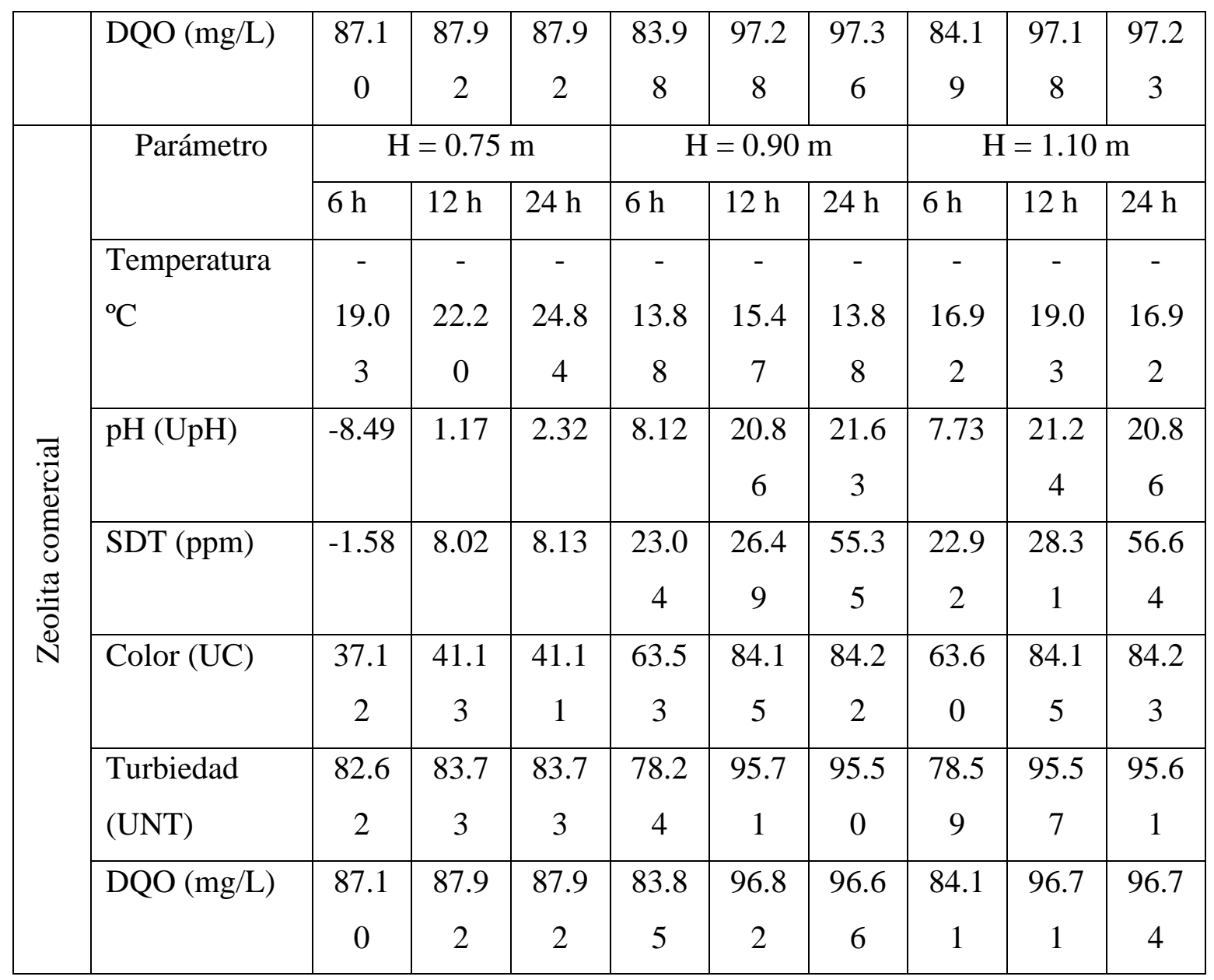

Fuente: Elaboración propia

\section{Discusión}

Los resultados muestran a la zeolita de cerro como el mejor medio de soporte comparada con la zeolita de río y la comercial (aunque presenta similitud con la zeolita comercial, que actualmente se utiliza en el tratamiento de aguas residuales). Lo atribuimos a las características físicas, químicas y mecánicas: la zeolita de cerro sobresalió en parámetros como la porosidad, absorción y humedad, determinantes en el tratamiento de aguas residuales. La altura (H) óptima en los BLFFA fue $0.9 \mathrm{~m}$, factor importante de evaluar ya que a mayor altura se aumentan los costos y las eficiencias no son relevantes para justificar la inversión y a menor altura cae el rendimiento en calidad del agua tratada. En ese sentido, las zeolitas nativas cuentan con propiedades físicas y químicas para ser utilizadas en el tratamiento de agua residual doméstica, tal y como plantea Florencia (2012), quien evaluó una zeolita nativa $\left(\mathrm{Fe}_{0.53} \mathrm{Ca}_{1.87} \mathrm{Mg}_{0.61} \mathrm{Na}_{0.93} \mathrm{~K}_{0.80} \mathrm{Si}_{29.00} \mathrm{Al}_{7.25} \mathrm{O}_{72}\right)$ en la remoción de nitrógeno 
Revista Iberoamericana de las Ciencias Biológicas y Agropecuarias

amoniacal y obtuvo hasta $78 \%$ con un TRH de 96 horas. Por otra parte, Hernández et al. (2010) identificaron que las zeolitas naturales de México provenientes de los yacimientos de Clinoptilolita y Mordenita tienen potencial de aplicación en tratamientos de aguas, biomedicina, agricultura y contaminación atmosférica, al igual que las zeolitas naturales de este estudio, que demostraron ser eficiente en el tratamiento de aguas residuales.

La mayoría de los procesos biológicos que en la actualidad se utilizan son lagunas de estabilización, lodos activados, biofiltros, biodiscos y la digestión anaerobia. Aunque estos ofrecen remociones aceptables de materia orgánica (> 80\%), una de sus desventajas es el TRH, que es demasiado grande. Mientras que en los BLFFA los TRH son mucho menores y permite que las bacterias estén en contacto con el alimento presente en las aguas residuales, el cual es suficiente para que estas asimilen o estabilicen la materia orgánica, pues si el TRH es muy pequeño no toda la materia orgánica será removida y el efluente tendrá valores altos de DQO, y si el TRH es muy grande no se garantiza buen desempeño (Conagua, 2016b). La meta esencial en los tratamientos biológicos tipo BLFFA es estabilizar la materia orgánica, coagular y remover los sólidos coloidales flotantes que se encuentran en las aguas, incluyendo en algunos casos la remoción de nutrientes como el nitrógeno y el fósforo (Noyola et al., 2013). La biomasa convierte la materia orgánica carbonácea disuelta y en estado coloidal en diferentes gases y tejidos celulares, formando capas biológicas compuestas de materia celular y de coloides orgánicos importantes en el medio de soporte en los BLFFA (Conagua, 2016b). En este experimento, los TRH de 24 horas ayudaron a una mayor eficiencia de remoción de DQO en las zeolitas de río y de cerro, lo que favoreció a una mayor presencia de microorganismos en el medio de soporte dentro de los BLFFA. También se ha demostrado que el TRH es importante en reactores de lecho fluidizado (lechos de perlas con $3.6 \mathrm{~mm}$ de $\varnothing)$, pues con TRH de 36 horas y con Methylobacterium sp (30-37 $\left.{ }^{\circ} \mathrm{C}\right)$ se favorece la degradación de compuestos tóxicos como formaldehído hasta en 98 \% (Qiu et al., 2014).

La temperatura juega un papel importante en el tratamiento de las aguas residuales pues acelera las reacciones bioquímicas, reduce la solubilidad de los gases e intensifica sabores y olores durante los tratamientos. Durante los experimentos se presentó una variación de $28.95^{\circ} \mathrm{C}$ a $30.02{ }^{\circ} \mathrm{C}$, lo que permitió el desarrollo y establecimiento de microorganismos mesófilos responsables de la degradación de la materia orgánica (Miao, Zhang, Jia, Liao y Li, 2018; Torres et al., 2020), cumpliendo con la NOM-001-SEMARNAT-1996 en el valor descarga, que es menor a $40{ }^{\circ} \mathrm{C}$. En cuanto al pH, este presentó variaciones en los 
Revista Iberoamericana de las Ciencias Biológicas y Agropecuarias

tratamientos presentándose de neutros a ligeramente alcalinos (de 7.3 a 8.9), condición importante para el establecimiento de microorganismos, y manteniendo el rango del criterio de descarga que establece la NOM-001-SEMARNAT-1996, que es 6.5 a 10 unidades. Espinosa, Delgado e Hidalgo (2020) trabajaron una planta de tratamiento de aguas residuales con un proceso combinado (un reactor anóxico, dos de mezcla completa aerobios y un reactor biológico de membrana) y con un alto contenido de nitrógeno. En cuanto a la temperatura, los comportamientos fueron de $38^{\circ} \mathrm{C}$ para el anóxico, $40{ }^{\circ} \mathrm{C}$ en los aerobios y $39^{\circ} \mathrm{C}$ para el de membrana, mientras el comportamiento del $\mathrm{pH}$ se mantuvo en ocho unidades en todos los procesos. Al comparar estos resultados con los nuestros, podemos observar que la temperatura es menor por estar sometida a un solo proceso como el lecho empacado y por las características del medio de soporte que son las zeolitas; en el caso del pH, el rango es ligeramente alcalino como en los procesos evaluados por Espinosa et al. (2020).

Los SDT son un parámetro que generalmente no se ocupa para medir el desempeño de los rectores, sin embargo, en este caso es importante determinar la capacidad de adsorción de iones disueltos. En este experimento se demostró que la zeolita de cerro es la más eficiente removiendo hasta $71.95 \%$. Los valores que se lograron disminuir son sales inorgánicas disueltas (Hadad, Maine y Bonetto, 2006). Con este desempeño se corrobora que este material pétreo de cerro es una zeolita que captura iones disueltos, pues, siguiendo a Mietto, Politeo, Breschigliaro y Borin (2015), para que materiales de soporte comunes (filtros biológicos o biofiltros) puedan tener valores similares a esta remoción se debe contar con TRH prolongados (> 6 días) para favorecer la eliminación de iones o nutrientes combinados con el efecto temperatura. Los valores de SDT en los efluentes de zeolita de cerro y la comercial de nuestro estudio están dentro de lo permitido para descarga en riego (Secretaria de Marina Recursos Naturales y Pesca [Semarnap]-Conagua-Secretaría de Desarrollo Urbano y Ecología [Sedue], 1989), con $266.63 \mathrm{mg} / \mathrm{L}$ y $424.40 \mathrm{mg} / \mathrm{L}$, respectivamente, ya que la concentración de SDT que no presentan efectos nocivos en ningún cultivo son menores a $500 \mathrm{mg} / \mathrm{L}$ y la zeolita de río sí presentó valores por arriba de este criterio con $805.20 \mathrm{mg} / \mathrm{L}$.

La turbiedad y color no son parámetros normados por la NOM-001-SEMARNAT1996, pero son utilizados como variables de medición rápida en el desempeño de los sistemas de tratamiento de aguas residuales y están directamente relacionados con los sólidos suspendidos (Ortiz, López, Torres y Pampillón, 2018; Torres et al., 2020). En ese sentido, el medio de soporte cumple una función filtrante de estos sólidos reteniéndolos por adhesión; y 
Revista Iberoamericana de las Ciencias Biológicas y Agropecuarias

en el caso de la materia coloidal, es estabilizada por la biomasa (Crites y Tchobanoglous, 2000), por lo que las zeolitas presentan eficiencias altas en la remoción de turbiedad y color ( $>91 \%$ y $>84 \%$ respectivamente). La zeolita de cerro ( $0.9 \mathrm{~m}$ de H y 24 horas de TRH) es la que alcanzó $96.44 \%$ de remoción de turbiedad y $84.41 \%$ de color.

La DQO cuantifica el oxígeno necesario para oxidar químicamente la materia orgánica (no putrescible) e inorgánica presente en el agua residual (O'Dell, 1993); se trata de la variable más importante en los procesos biológicos para medir el desempeño de los sistemas de tratamiento de aguas residuales (Crites y Tchobanoglous, 2000). Espinosa et al. (2020) evaluaron un sistema de tratamiento biológico (anóxico-aerobio-reactor biológico de membrana) que opera una empresa dedicada a la elaboración de harinas de sardina y atún, cuyos afluentes se encuentran muy altos en DQO $(7728.9 \pm 513.8 \mathrm{mg} / \mathrm{L})$ y los efluentes $(353.8 \pm 62.1 \mathrm{mg} / \mathrm{L})$ se encuentran por arriba del proyecto de norma PROY-NOM-001SEMARNAT-2017 (Semarnat, 5 de enero de 2018) para descarga en zonas marinas y estuarios ( $85 \mathrm{mg} / \mathrm{L}$ de DQO), sin embargo la eficiencia de remoción de este sistema se reporta alta en $95.4 \%$ para este parámetro. En los tratamientos evaluados en este experimento se puede observar que la zeolita de cerro $(97.36 \%$ ) y comercial $(96.66 \%)$ tienen mucha similitud en la remoción de DQO con 24 horas de retención y 0.9 de H; la que presenta la menor eficiencia es la zeolita de río (93.71\%). Sin embargo, en nuestro caso, al estar tratando aguas residuales domésticas, el influente presenta concentración promedio de $373 \mathrm{mg} / \mathrm{L}$, lo que permite que los efluentes (9.8 mg/L de DQO) cumplan los límites permisibles de PROYNOM-001-SEMARNAT-2017 (Semarnat, 5 de enero de 2018).

Comparando las zeolitas de este estudio con otros medios de soporte y ambientes anaerobios que se han utilizado en biorreactores de lecho fijo o similares, podemos establecer que la zeolita de cerro tienen un alto potencial para su uso en las aguas residuales por las altas eficiencias de remoción obtenidas. Rivas et al. (2003) alcanzaron $91 \%$ de remoción con cargas de DQO iniciales de $463 \mathrm{mg} / \mathrm{L}$, muy similares a las de nuestro estudio (agua doméstica); Castillo et al. (2006), con material de desecho de mazorcas como medio de soporte, registraron remociones entre 50 y $70 \%$ de DQO de agua residual y agua porcina; Rodríguez et al. (2007), utilizando un medio de soporte cerámico, registraron $57 \%$ de remoción de DQO de un agua residual doméstica; Cárdenas y Ramos (2009) encontraron que las conchas marinas permiten alcanzar hasta 89.7 \% de remoción de DQO. Finalmente, este tratamiento experimental con BLFFA presenta desempeños similares (> $93 \%$ de DQO) a los 
procesos aerobios de biomasa en suspensión como los reactores discontinuos secuenciales reportados por Torres et al. (2020), donde su mejor tratamiento se da con un TRH de 20 horas, que alcanza $92.4 \%$ de remoción de DQO. Sin duda esto muestra que el TRH es determinante en la remoción por tiempo de contacto de los microorganismos con el agua residual.

\section{Conclusión}

Las zeolitas nativas de cerro y de río deben ser implementadas en el tratamiento de aguas residuales domésticas en BLFFA pues son eficientes en la remoción de contaminantes básicos y favorece los tiempos y costos de operación.

Los experimentos muestran que, aunque no existe una diferencia estadística significativa entre el uso de 12 o 24 horas de TRH de 20 horas en la remoción de color, turbiedad y DQO, las mejores eficiencias de remoción para estos parámetros se alcanzan en 24 horas de TRH.

La zeolita de cerro es la que presenta el mejor rendimiento en la remoción de contaminantes básicos operando con $0.9 \mathrm{~m}$ de $\mathrm{H}$ y con 24 horas de TRH.

Para la utilización de los BLFFA es necesario que previo al proceso se cuente con un sistema de pretratamiento para la eliminación de sólidos sedimentables y suspendidos.

Finalmente, podemos recomendar la implementación del uso de los BLFFA para el tratamiento secundario de efluentes domésticos en sistemas descentralizados del sureste de México como una alternativa viable en el tratamiento de sus aguas residuales.

\section{Futuras líneas de investigación}

Para conocer el potencial de las zeolitas nativas en reactores de lecho empacado se debe continuar el estudio en los reactores en un corto plazo y evaluar si los tiempos de retención hidráulica con la operación actual son suficientes para poder remover nutrientes (nitrógeno y fósforo) y otros xenobióticos en valores de concentración que permitan descargar a cuerpos receptores de forma segura, como lo establece el marco normativo ambiental vigente. Sabemos que es necesario configurar un tren de tratamiento a la medida del tipo de agua a procesar, por lo que otra línea de investigación a mediano plazo sería evaluar la eficiencia total de un tren de tratamiento piloto-experimental con aguas de 
concentración media y fuerte, con diferentes configuraciones en el tren (secundario) con unidades en serie, aerobio, anaerobio, etc., de manera tal que se pueda recomendar un tren de tratamiento para diferentes casos eficiente y económico.

\section{Agradecimientos}

La autora principal agradece al Tecnológico Nacional de México por gestionar en tiempo y forma la beca-comisión y el apoyo financiero del Programa para el Desarrollo Profesional Docente (Prodep) y así contar con el tiempo suficiente para la realización de esta investigación. De igual manera, se agradece a la Universidad Juárez Autónoma de Tabasco y particularmente a la DACBiol por el apoyo incondicional brindado en el Laboratorio de Tecnología del Agua y a sus gestores, Dr. Gaspar López Ocaña y Dr. Raúl Germán Bautista Margulis, por todas las facilidades para la realización de este proyecto.

\section{Referencias}

Cárdenas, G. L. y Ramos, R.M. (2009). Evaluación de la eficiencia de reactores de lecho fijo utilizando aguas mieles residuales de trapiches artesanales. Ciencia e Ingeniería Neogranadina, 19(1), 25-38.

Castillo, E. F., Solano, J. K. y Rangel, M. P. (2006). Evaluación operacional de un sistema a escala laboratorio de biopelícula anaerobia soportada para el tratamiento de aguas residuales domésticas. Revista ION, 19(1): 18-22.

Comisión Nacional del Agua [Conagua]. (2016a). Manual de agua potable, alcantarillado y saneamiento. Introducción al tratamiento de aguas residuales municipales. México: Secretaría de Medio Ambiente y Recursos Naturales, Comisión Nacional del Agua, Subdirección General de Agua Potable, Drenaje y Saneamiento. Recuperado de https://files.conagua.gob.mx/conagua/mapas/SGAPDS-1-15-Libro25.pdf.

Comisión Nacional del Agua [Conagua]. (2016b). Diseño de plantas de tratamiento de aguas residuales municipales. Manual de agua potable, alcantarillado y saneamiento: procesos de oxidación bioquímica con biomasa fija. México: Secretaría de Medio Ambiente y Recursos Naturales, Comisión Nacional del Agua, Subdirección General de Agua Potable, Drenaje y Saneamiento. Recuperado de https://files.conagua.gob.mx/conagua/mapas/SGAPDS-1-15-Libro34.pdf. 
Crites, R. y Tchobanoglous, G. (2000). Sistemas de manejo de aguas residuales para núcleos pequeños y descentralizados. Colombia: McGraw-Hill.

Environmental Protection Agency [EPA]. (1983). Turbidity (Nephelometric) Methods for Chemical Analysis of Water and Wastes. Environmental Monitoring and Supporting Laboratory. Cincinnati, United States: Environmental Protection Agency.

Espinosa, M. A., Delgado, D. R. e Hidalgo, M. A. (2020). Evaluación de un proceso anóxicoaerobio-reactor biológico de membrana con alto contenido de nitrógeno. Revista Internacional de Contaminación Ambiental, 36(2), 303-320.

Fernández, M., Flores, D., Yactayo, M., Lovera, D., Quispe, J., Landauro, C. y Pardave, W. (2020). Remoción de metales pesados desde efluentes mineros, mediante cáscaras de frutas. Aibi, Revista de Investigación, Administración e Ingeniería, 8(1), 21-28.

Florencia, M. (2012). Estudio de zeolitas procedentes de depósitos argentinos. Aspectos tecnológicos que posibiliten su aplicación en agroindustria y contralor ambiental. (Tesis doctoral). Universidad Nacional de La Plata, La Plata. Recuperado de https://1library.co/document/nq7rg3dy-procedentes-depositos-argentinos-aspectostecnologicos-posibiliten-aplicacion-agroindustria.html.

Ganesh, R., Rajinikanth, R., Thanikal, J. V., Ramanujam R. A. and Torrijos, M. (2010). Anaerobic treatment of winery wastewater in fixed bed reactors. Bioprocess and Biosystems Engineering, 33, 619-628. Retrieved from https://link.springer.com/article/10.1007/s00449-009-0387-9.

Guerrero, M. S., Vázquez, A. y Rodríguez, M. (2019) La zeolita en la descontaminación de aguas residuales. Universidad, Ciencia y Tecnología, 21(2), 109-117.

Hadad, H. R., Maine, M. A. and Bonetto, C. A. (2006). Macrophyte growth in a pilot scale constructed wetland for industrial wastewater treatment. Chemosphere, 63(10), 17441753.

Hernández, M.A., Rojas, F., Lara, V.H., Portillo, R., Castelán, R., Pérez, G. y Salas, R. (2010). Estructura porosa y propiedades estructurales de mordenita y clinoptilolita. Superficies y Vacío, 23, 51-56.

Miao, Y., Zhang, X. X., Jia, S., Liao, R. and Li, A. (2018). Comprehensive analyses of functional bacteria and genes in a denitrifying EGSB reactor under $\mathrm{Cd}(\mathrm{II})$ stress. Applied Microbiology and Biotechnology, 102, 8551-8560. Retrieved from https://doi.org/10.1007/s00253-018-9228-6. 
Mietto, A., Politeo, M., Breschigliaro, S. and Borin, M. (2015). Temperature influence on nitrogen removal in a hybrid constructed wetland system in northern italy. Ecological Engineering, 75, 291-302.

Morató J., Pires, A. y Subirana, A. (2009). Crisis del agua. En Peñuelo, G. y Morató, J. (eds.), Manual de tecnologías sostenibles en tratamiento de aguas (pp. 13-26). Tecnologías Sostenibles para la Potabilización y el Tratamiento de Aguas Residuales.

Muñoz, D. J., Soler, A., López, F. y Hernández, M. M. (2015). Edafología: manual de métodos de análisis del suelo. México: Universidad Nacional Autónoma de México.

Noyola A., Morgan J. M. y Güereca L. P. (2013). Selección de tecnologías para el tratamiento de aguas residuales municipales. Guía de apoyo para ciudades pequeñas y medianas (1. ${ }^{\mathrm{a}}$ ed.). México: Universidad Nacional Autónoma de México.

O’Dell, J. W. (ed.) (1993). Method 410.4, Revision 2.0: The Determination of Chemical Oxygen Demand by SemiAutomated Colorimetry. Cincinnati, United States: Environmental Protection Agency. Retrieved from https://www.epa.gov/sites/production/files/2015-08/documents/method_4104_1993.pdf.

Organización de las Naciones Unidas para la Educación, la Ciencia y la Cultura [Unesco]. (2017). Informe mundial de las Naciones Unidas sobre el desarrollo de los recursos hídricos, 2017: Aguas residuales: el recurso no explotado. París, Francia: Organización de las Naciones Unidas para la Educación, la Ciencia y la Cultura. Recuperado de http://www.unesco.org/new/es/naturalsciences/environment/water/wwap/wwdr/2017-wastewater-the-untapped-resource/.

Ortiz, V., López, G., Torres, A. y Pampillón, L. (2018). Almidón de yuca (Manihot esculenta Crantz) como coadyuvante en la coagulación-floculación de aguas residuales domésticas. Revista Iberoamericana de las Ciencias Biológicas y Agropecuarias, 7(13), 18-46.

Pérez, T., Pereda, I., Teixeira, G., Pozzi, E., Hong, W., Oliva, D. y Zaiat, M. (2019). Adición de zeolita en reactor EGSB para el tratamiento de aguas residuales porcinas. Infomin, 11. Recuperado de http://www.infomin.co.cu/index.php/i/article/view/120.

Qiu, L., Chen, W., Zhong, L., Wu, W., Wu, S., Chen, J., Zhang, F. and Zhong, W. (2014). Formaldehyde biodegradation by immobilized Methylobacterium sp. XJLW cells in 
a three-phase fluidized bed reactor. Bioprocess and Biosystems Engineering, 37, 1377-1384.

Rivas, B. A., Nevárez, G. V., Bautista, R. G., Pérez, A. y Saucedo, R. (2003). Tratamiento de aguas residuales de uso agrícola en un biorreactor de lecho fijo. Agrociencia, 37(2), 157-166. Recuperado de http://www.redalyc.org/articulo.oa?id=30237206.

Rodríguez, T., Pinzón, L. y Arámbula, C. (2007). Implementación de un biorreactor de lecho empacado cerámico para el tratamiento de aguas residuales domésticas. En Restrepo, I., Sánchez, L. D., Galvis, A., Rojas, J. y Sanabria, I. J. (comps.), Avances en investigación y desarrollo en agua y saneamiento para el cumplimiento de las metas del milenio (pp. 210-217). Santiago de Cali, Colombia: Programa Editorial Universidad del Valle. Recuperado de https://books.google.es/books?hl=es\&lr=\&id=vpFqgpfHBmYC\&oi=fnd\&pg=PA21 $0 \& d q=i m p o r t a n c i a+d e+l e c h o+e m p a c a d o \& o t s=M 22 S t j 6 X C J \& s i g=H e h M i s j y I Y d 6 j s$ CxbtfN_XcAdZc\#v=onepage\&q=importancia\%20de\%20lecho\%20empacado\&f $\mathrm{f}=\mathrm{fal}$ se.

Secretaría de Marina Recursos Naturales y Pesca [Semarnap]-Comisión Nacional del Agua [Conagua]-Secretaría de Desarrollo Urbano y Ecología [Sedue]. (13 de diciembre de 1989). Criterios ecológicos de calidad del agua CE-CCA-001/89. Diario Oficial de la Federación.

Secretaría de Medio Ambiente y Recursos Naturales [Semarnat]. (31 de diciembre de 2002). Norma Oficial Mexicana NOM-021-RECNAT-2000, la cual establece las especificaciones de fertilidad, salinidad y clasificación de suelos, estudios, muestreo y análisis. Diario Oficial de la Federación. Recuperado de http://www.ordenjuridico.gob.mx/Documentos/Federal/wo69255.pdf

Secretaría de Medio Ambiente y Recursos Naturales [Semarnat]. (5 de enero de 2018). Proyecto de Modificación de la Norma Oficial Mexicana NOM-001-SEMARNAT1996, que establece los límites máximos permisibles de contaminantes en las descargas de aguas residuales en aguas y bienes nacionales para quedar como proyecto de modificación de la Norma Oficial Mexicana PROY-NOM-001SEMARNAT-2017, que establece los límites permisibles de contaminantes en las descargas de aguas residuales en cuerpos receptores propiedad de la nación. Diario 
Revista Iberoamericana de las Ciencias Biológicas y Agropecuarias

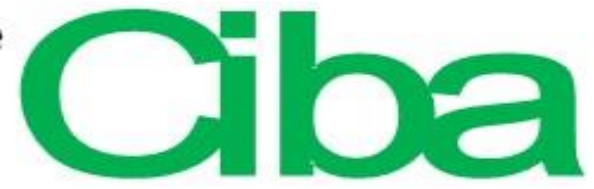

Oficial

de

la

Federación.

Recuperado

de https://www.dof.gob.mx/nota_detalle.php?codigo=5510140\&fecha=05/01/2018.

Secretaría de Medio Ambiente y Recursos Naturales [Semarnat]-Comisión Nacional del Agua [Conagua]. (s. f.). Normas Oficiales Mexicanas. NOM-001-SEMARNAT-1996. NOM-002-SEMARNAT-1996. NOM-003-SEMARNAT-1997. México: Secretaría de Medio Ambiente y Recursos Naturales-Comisión Nacional del Agua. Recuperado de http://www.conagua.gob.mx/CONAGUA07/Publicaciones/Publicaciones/SGAA15-13.pdf.

Standard Methods For the Examination of Water and Wastewater. (2017). 2120 Color. Washington, United States: American Public Health Association. Retrieved from https://www.standardmethods.org/doi/10.2105/SMWW.2882.017.

Standard Methods For the Examination of Water and Wastewater. (2017). 2540 Total Dissolved Solids. Washington, United States: American Public Health Association. Retrieved from https://www.standardmethods.org/doi/abs/10.2105/SMWW.2882.027.

Standard Methods For the Examination of Water and Wastewater. (2017). 2550 Temperature.

Washington, United States: American Public Health Association. Retrieved from https://www.standardmethods.org/doi/abs/10.2105/SMWW.2882.031.

Standard Methods For the Examination of Water and Wastewater. (2017). 4500-H+ pH Value. Washington, United States: American Public Health Association. Retrieved from https://www.standardmethods.org/doi/10.2105/SMWW.2882.082.

Tchobanoglous, G., Burton, F. and Stensel, H. (2003). Wastewater Engineering. New York, United States: Metcalf \& Eddy Inc.

Torres, C. A., López, G., Romellón, M. J., Vazquez, M. B. y Comparán, L. E. (2018). Biomasa de origen vacuno en la remoción de contaminantes básicos en un reactor discontinuo secuencial. Revista Iberoamericana de las Ciencias Biológicas y Agropecuarias, 9(18), 1-32.

Vian, J., Vigueras, S. E., Velasco, A. and Puebla, H. (2020). A Novel Up-Flow Anaerobic Sludge Blanket Solid-State Reactor for the Treatment of Fruit and Vegetable Waste. Environmental Engineering Science, 37(5). Retrieved from https://doi.org/10.1089/ees.2019.0369. 
Vigueras, S., Vian, J., Velasco, A. y Zafra, G. (2016) Diseño y operación de un reactor anaerobio de flujo ascendente empacado con lecho de lodos (RAFAELL) para la producción de metano. Revista de Sistemas Experimentales, 3(8), 1-7.

\section{Noemi Méndez de los Santos}

Candidata a doctora en Ciencias en Ecología y Manejo de Sistemas Tropicales, maestro en Ingeniería y Protección Ambiental e ingeniero Civil, todos los grados por la Universidad Juárez Autónoma de Tabasco (UJAT). Es profesora-investigadora de tiempo completo en el Instituto Tecnológico de Villahermosa.

\section{Gaspar López Ocaña}

Doctor en Ciencias en Ecología y Manejo de Sistemas Tropicales, maestro en Ingeniería y Protección Ambiental e ingeniero Ambiental, todos los grados por la Universidad Juárez Autónoma de Tabasco (UJAT). Es profesor-investigador de tiempo completo, miembro del cuerpo académico "Ingeniería y tecnología ambiental" y responsable del Laboratorio de Tecnología del Agua de la División Académica de Ciencias Biológicas (DACBiol) de la UJAT. 


\begin{tabular}{|c|c|}
\hline Rol de contribución & Autor(es) \\
\hline Conceptualización & Noemi Méndez de los Santos $50 \%$, Gaspar López Ocaña $50 \%$ \\
\hline Metodología & Noemi Méndez de los Santos $50 \%$, Gaspar López Ocaña $50 \%$ \\
\hline Software & Gaspar López Ocaña 100 \% \\
\hline Validación & Noemi Méndez de los Santos $50 \%$, Gaspar López Ocaña 50\% \\
\hline Análisis formal & Noemi Méndez de los Santos $50 \%$, Gaspar López Ocaña 50\% \\
\hline Investigación & Noemi Méndez de los Santos $80 \%$, Gaspar López Ocaña $20 \%$ \\
\hline Recursos & Noemi Méndez de los Santos $80 \%$, Gaspar López Ocaña $20 \%$ \\
\hline Curación de datos & Noemi Méndez de los Santos $20 \%$, Gaspar López Ocaña $80 \%$ \\
\hline $\begin{array}{l}\text { Escritura. Preparación del } \\
\text { borrador original }\end{array}$ & Noemi Méndez de los Santos 50 \%, Gaspar López Ocaña $50 \%$ \\
\hline Escritura. Revisión y edición & Noemi Méndez de los Santos $50 \%$, Gaspar López Ocaña 50 \% \\
\hline Visualización & Noemi Méndez de los Santos $50 \%$, Gaspar López Ocaña $50 \%$ \\
\hline Supervisión & Noemi Méndez de los Santos $50 \%$, Gaspar López Ocaña 50 \% \\
\hline Administración de proyectos & Noemi Méndez de los Santos $50 \%$, Gaspar López Ocaña $50 \%$ \\
\hline Adquisición de fondos & Noemi Méndez de los Santos $100 \%$ \\
\hline
\end{tabular}

